\title{
Gas mixing enhanced by power modulations in atmospheric pressure microwave plasma jet
}

\author{
J Voráć ${ }^{1}$, L Potočňáková ${ }^{1}$, P Synek ${ }^{2}$, J Hnilica ${ }^{1}$ and V Kudrle ${ }^{1}$ \\ ${ }^{1}$ Department of Physical Electronics, Masaryk University, Kotlářská 2, CZ-61137 Brno, Czech Republic \\ 2 Group of plasma technologies, CEITEC: Central European Institute of Technology, Masaryk University, \\ Kamenice 753/5, CZ-62500 Brno, Czech Republic
}

E-mail: vorac@mail.muni.cz

Received 9 July 2015, revised 4 January 2016

Accepted for publication 26 January 2016

Published 29 February 2016

\begin{abstract}
Microwave plasma jet operating in atmospheric pressure argon was power modulated by audio frequency sine envelope in the $10^{2} \mathrm{~W}$ power range. Its effluent was imaged using interference filters and ICCD camera for several different phases of the modulating signal. The combination of this fast imaging with spatially resolved optical emission spectroscopy provides useful insights into the plasmachemical processes involved. Phase-resolved schlieren photography was performed to visualize the gas dynamics. The results show that for higher modulation frequencies the plasma chemistry is strongly influenced by formation of transient flow perturbation resembling a vortex during each period. The perturbation formation and speed are strongly influenced by the frequency and power variations while they depend only weakly on the working gas flow rate. From application point of view, the perturbation presence significantly broadened lateral distribution of active species, effectively increasing cross-sectional area suitable for applications.
\end{abstract}

Keywords: microwave plasma, atmospheric pressure, plasma jet, gas mixing, vortex

S Online supplementary data available from stacks.iop.org/PSST/25/025018/mmedia

(Some figures may appear in colour only in the online journal)

\section{Introduction}

Low temperature atmospheric pressure plasma discharges are widely used in many technological applications such as plasma surface treatment of various materials, plasma enhanced chemical vapour deposition (PECVD) of thin films, pollutant degradation, natural gas reforming or synthesis of advanced materials. Besides these, the usage of atmospheric plasma sources in medical and biomedical applications was studied recently. Examples may be plasma medicine, accelerated wound healing, disinfection and decontamination of both living tissue and medical instruments [1-15].

The atmospheric pressure plasma jets are significantly nonuniform in both axial and radial direction [16]. The greatest change of plasma properties takes place near the discharge tube end where the plasma plume mixes with ambient atmosphere. This mixing zone is very important as many active species necessary for the plasma surface treatment (or other applications) are actually formed there. The spatial profile of their concentration is affected by many parameters such as composition of working gas, its flow rate, discharge tube diameter, configuration of the plasma excitation, input power, current state of the ambient atmosphere, etc.

Plasma jets, with their steep gradients of physical quantities, local inhomogeneities, flow turbulences, temporal instabilities and strongly interconnected electromagnetics, fluid dynamics, plasma physics, and plasma chemistry [17-22] are notoriously difficult to be fully theoretically described. Existing theories and models [23-27] therefore always employ suitable approximations and simplifications and are 


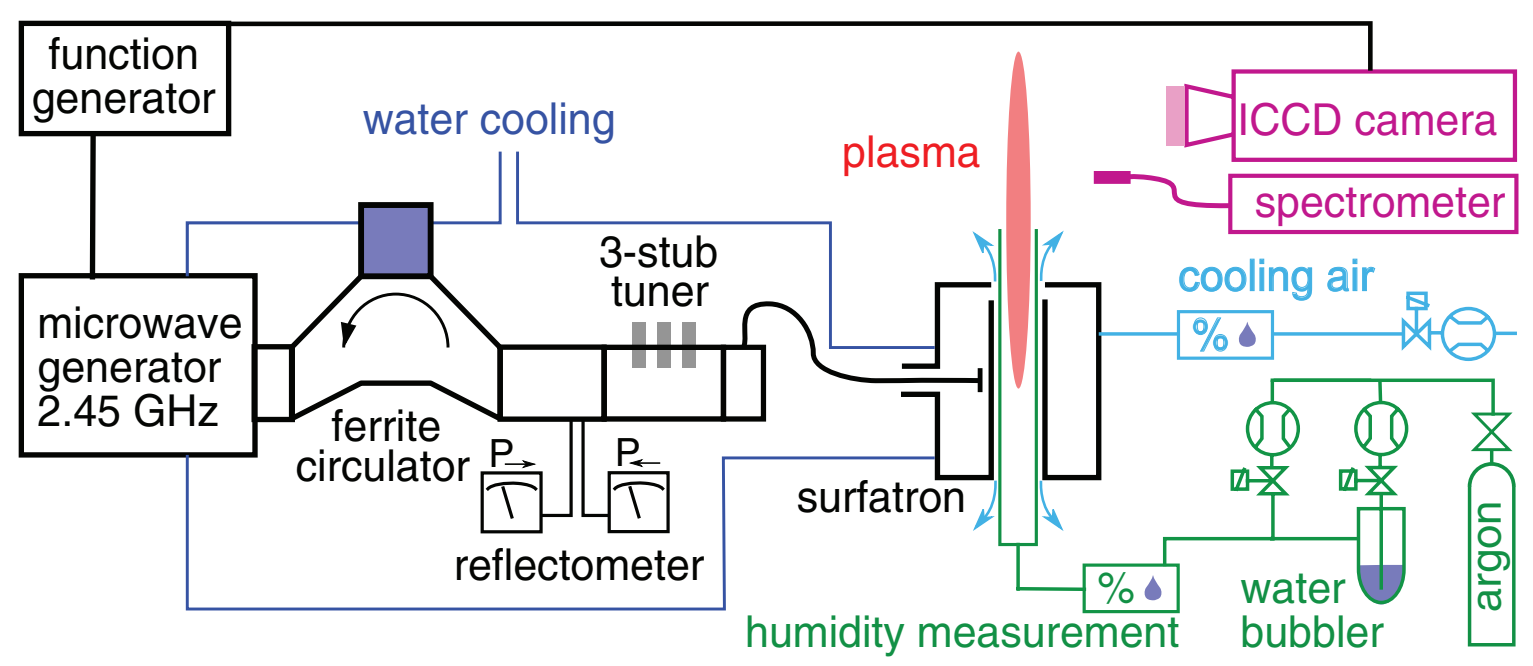

Figure 1. Schematic drawing of the experimental set-up.

validated by comparison with comprehensive experimental observations.

Recent experimental investigations of the plasma jets were carried out using advanced diagnostic techniques, such as high speed intensified charge-coupled device (ICCD) imaging, shadowgraphy, schlieren visualization or planar laser-induced fluorescence (PLIF) [21, 22, 26, 28-32]. These methods can provide excellent spatio-temporal resolution and are able to reveal the complex fluid dynamics i.e. laminar and turbulent gas flow regimes or the formation of transient vortex structures.

Advantageously using inherent plasma non-linearities, the discharges excited using pulsed or modulated power can have significantly lower thermal loading of the substrate with higher density of active particles (such as electrons, ions, excited species, atoms, molecules, radicals, etc) than the continuous wave plasma at the same mean power [33-37]. Moreover, the modulation offers additional tunable parameters, which enables better optimisation of the plasma process [38-40].

Although the modulations are most often of the simple on/off variant, more complex modulation schemes are possible. From the point of view of frequency domain analysis, the simplest modulation of all is the modulation by sine wave. Some aspects of the influence of such modulation on atmospheric plasma jet were published recently [41-44] and were found suitable for various applications [9, 45, 46]. Among others, effective broadening of the active plasma region was observed for certain power modulation frequencies [42].

In this paper, the plume of power modulated plasma jet is studied by means of high speed ICCD imaging in phasesynchronized mode using spectral bandpass filters, which discern the regions of various dominant spectral emission activity in the plume. When combined with time-averaged standard optical emission spectroscopy using a spectrometer it can reveal and visualize complex fluid dynamics and plasma-chemical processes that govern the unstable plasmaambient interface. These observations are supplemented by phase-resolved schlieren photography which reveals some aspects of the gas dynamics.

\section{Experimental set-up}

The whole experimental set-up can be seen in figure 1. It consists of the plasma device itself and diagnostic devices.

\subsection{Plasma system}

The principal component is a particular type of microwave plasma jet - the surfatron (SAIREM Surfatron 80). The power to this surface wave driven jet [47] was supplied by microwave (2.45 GHz) generator (SAIREM GMP 20 KED) in amplitude modulated (AM) mode, for which, if not said otherwise, sine envelope modulation with $165 \mathrm{~W}$ in minimum and $315 \mathrm{~W}$ in maximum of output power at modulation frequencies $90 \mathrm{~Hz}$, $900 \mathrm{~Hz}$ or $1710 \mathrm{~Hz}$ was used. The microwaves were fed from the magnetron to the surface wave launcher via a waveguide, ferrite circulator, reflectometer, 3-stub tuner and coaxial cable. Impedance matching was adjusted specifically for each modulation frequency to achieve less than $3 \%$ reflected power at the mean power while keeping the reflected power below $10 \%$ at power maxima and minima.

In figure 2 the actual microwave power envelopes are shown. For $90 \mathrm{~Hz}$ and $900 \mathrm{~Hz}$ the power envelope matches the modulating sine signal but for the highest modulation frequency of $1710 \mathrm{~Hz}$, the actual output power envelope is visibly distorted. This can be attributed to a limited frequency response and/or slew rate of the voltage controlled magnetron supply.

The plasma is excited inside a fused silica discharge tube ( $2 \mathrm{~mm}$ inner diameter, $4 \mathrm{~mm}$ outer one) vertically passing through the centre of the surfatron body, the tube ending $1 \mathrm{~cm}$ above the launching gap. Atmospheric pressure argon (1 slm) with small admixture of water vapour $(2600 \mathrm{ppm}(\mathrm{V}))$ is used as a working gas flowing through the discharge tube. The water is added intentionally to obtain reproducible humidity, since the water vapour is always present in the feed gas, unless extensive effort is made to remove it. The discharge tube is open into ambient atmosphere, where the argon plasma effluent mixes with air and where a possible material treatment 


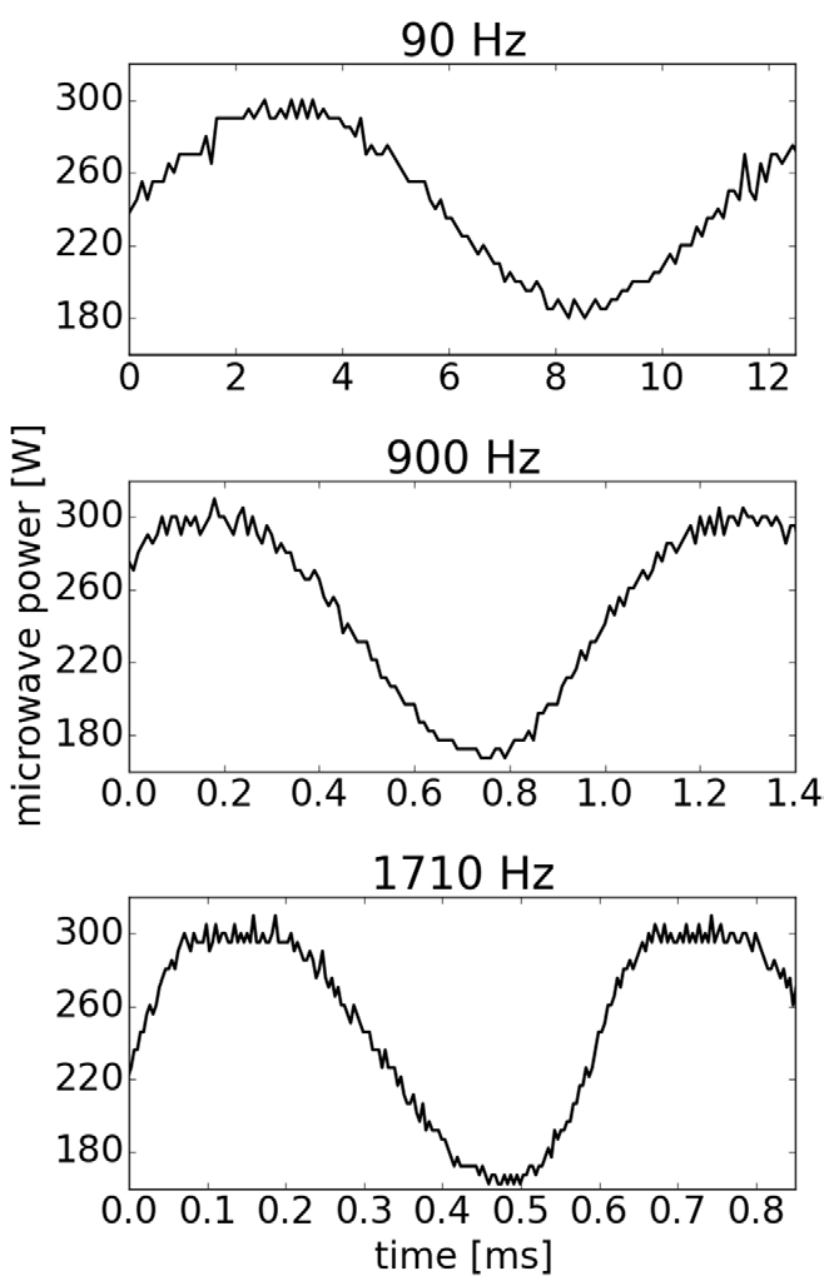

Figure 2. Actual magnetron output power envelopes for three modulating frequencies. Although the modulating signal was sine in all cases, the resulting power envelope is for $1710 \mathrm{~Hz}$ distorted.

would take place. This plasma plume is the main focus of this paper.

The generator, the circulator and the surfatron body are cooled by water. Additionally, the discharge tube itself is cooled by externally flowing compressed dry air $(5 \mathrm{slm})$ with controlled humidity $(5000 \mathrm{ppm}(\mathrm{V}))$.

In the jet plume, there are thus three interacting gas environments: (i) the argon plasma, outflowing from the discharge tube, with elevated gas temperature (in the order of several thousand Kelvin [43]) and humidity of $2600 \mathrm{ppm}(\mathrm{V})$, (ii) cooling air at temperature lower than that in the discharge, flowing co-axially around the plasma jet, forming a gas shield around a part of the plume, with humidity around 5000 $\operatorname{ppm}(\mathrm{V})$, and (iii) the stationary ambient air at room temperature and humidity in the order of $10000 \mathrm{ppm}(\mathrm{V})$. The whole experiment was encompassed by plastic sheet to minimize any unwanted perturbations of the jet and its surroundings from outside.

\subsection{Optical diagnostics}

The ICCD camera (PI-MAX2 1024RB-25-FG43, 16-bit greyscale resolution, $256 \times 1024$ pixels) equipped with macro lens is observing the whole discharge above the surfatron body (i.e. $1 \mathrm{~cm}$ inside the discharge tube + several $\mathrm{cm}$ of the plasma effluent and the plasma afterglow above the tube) through following bandpass filters (the given widths are FWHM):

$(248 \pm 5) \mathrm{nm}$ corresponding to $\mathrm{NO} \gamma$ system, i.e. the bands of NO $\left(A^{2} \Sigma^{+} \rightarrow X^{2} \Pi\right)$ transition

$(307 \pm 10) \mathrm{nm}$ corresponding to $\mathrm{OH}\left(A^{2} \Sigma^{+} \rightarrow X^{2} \Pi\right)$ transition, namely the vibration band $(0,0)$ and, to a limited extent, also $(1,1)^{3}$. Nitrogen $2^{\text {nd }}$ positive system, vibration bands $(2,1),(3,2)$ and $(4,3)$ may also be present.

$(355 \pm 10) \mathrm{nm}$ corresponding to $2^{\text {nd }}$ positive system of nitrogen $\left(\mathrm{N}_{2} \quad\left(C^{3} \Pi_{u} \rightarrow B{ }^{3} \Pi_{g}\right)\right.$ transition), vibration bands $(0,1),(1,2)$ and $(2,3)$. In this wavelength range, the $1^{\text {st }}$ negative system $\mathrm{N}_{2}^{+}\left(B^{2} \Sigma_{u}^{+} \rightarrow X^{2} \Sigma_{g}^{+}\right)$, vibration bands satisfying $(v, v-1)$ with $v=1,2,3,4,5$, is also present.

(780 \pm 10$) \mathrm{nm}$ corresponding to argon transitions $2 p_{2} \rightarrow 1 s_{3}{ }^{4}$ at $772.42 \mathrm{~nm}$ and $2 p_{7} \rightarrow 1 s_{5}$ at $772.37 \mathrm{~nm}$ and oxygen triplet $3 p^{5} P_{1,2.3} \rightarrow 3 s^{5} S_{2}$ around $777.4 \mathrm{~nm}$

$(845 \pm 5) \mathrm{nm}$ corresponding to argon transitions $2 p_{8} \rightarrow 1 s_{4}$ at $842.47 \mathrm{~nm}$ and $2 p_{4} \rightarrow 1 s_{2}$ at $852.14 \mathrm{~nm}$ and oxygen triplet $3 p^{3} P_{0,1,2} \rightarrow 3 s^{3} S_{1}$ at $844.6 \mathrm{~nm}$

The transmittances of the optical filters as measured by spectrophotometer PerkinElmer Lambda 1050 are shown in figure 5.

In order to achieve phase resolved measurements, the ICCD camera is triggered by the same signal which was modulating the microwave generator with variable delay. For each modulation frequency, more than one full period was captured with at least 24 time-steps. The lens aperture was set to minimum to obtain depth of field larger than the discharge diameter. Nevertheless, the objective lens had to be slightly refocused for each bandpass filter. To compensate for this change in field-of-view, the recorded images are resized by 2D interpolation.

Additionally, the time-integrated overview optical emission spectra of the discharge at various axial positions are obtained using a fixed grating spectrometer (Avantes AvaSpec-2048TEC-2) with $1.2 \mathrm{~nm}$ resolution. The geometry (optic fibre aperture, working distance) gives spatial resolution around $1 \mathrm{~mm}$.

\subsection{Phase-resolved schlieren photography}

The schlieren photography was performed in the single mirror configuration, as can be seen in figure 3. As a light source, high-power single chip blue light-emitting diode (LED) emitting predominantly at $465 \mathrm{~nm}$ was used, with a pinhole aperture placed into direct contact with the diode. In such way, the point light source of sufficient brightness and uniformity was achieved. The light source was positioned at the axis of the spherical mirror, two focal lengths away from it, so that the focused point image is projected in the same plane as the light source. The razor edge, used to block the image, was positioned vertically. The camera capturing the deviated light

\footnotetext{
${ }^{3}$ This notation shows the vibrational auantum number of upper $\left(v^{\prime}\right)$ and lower $\left(v^{\prime \prime}\right)$ state, respectively, as $\left(v^{\prime}, v^{\prime \prime}\right)$.

${ }^{4}$ The argon states are labelled in Paschen's notation.
} 


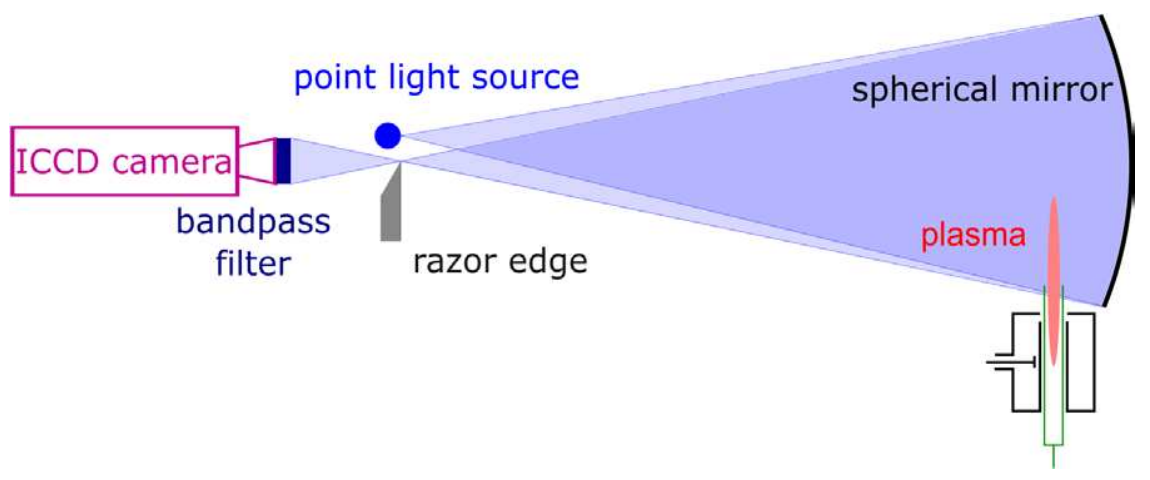

Figure 3. Schematic drawing of the phase-resolved schlieren photography set-up.

passing beyond the edge is the same ICCD camera as was used for optical diagnostics with the same triggering system. The discharge itself was placed into the close proximity of the mirror. The mirror thus did not form a real image of the discharge and the emission of the plasma appears at the ICCD camera only as a background noise. To minimize the amount of this diffused light from the plasma, a bandpass filter was used. The filter with central wavelength of $462.5 \mathrm{~nm}$ and FWHM of $5 \mathrm{~nm}$ was chosen, as most of the LED light could pass through, yet the discharge emitted only insignificantly at this wavelength.

\section{Results and discussions}

\subsection{Overview spectra}

To identify the significant features in the emission spectrum of the discharge plume, the time-integrated overview spectra were recorded at various axial positions, see figure 4 .

The lowermost spectrum is the emission from the discharge tube interior, which should contain mainly the feed gas constituents, i.e. argon and water. At the discharge tube end, the mixing with ambient atmosphere starts, as evidenced by $\mathrm{NH}$ band. In the plasma plume, the nitrogen emissions are very strong while argon intensities are falling. In the afterglow region the argon is de-excited and so the emission is dominated by long living excited species and products of their reactions.

For the following discussion, more detailed view (see figure 5) of important spectral regions is needed. Starting from the interior of the discharge tube, the dominant emission lines in the region between 700 and $850 \mathrm{~nm}$, are those of argon and atomic oxygen. The argon lines are getting weaker with the distance from the surfatron cavity. The energy of the upper states of argon is around $13 \mathrm{eV}$ and the lines appear due to the transition to lower states with energy around $11.5 \mathrm{eV}$. Two of these lower states are metastable with radiative lifetime in the order of seconds, see e.g. [48]. The argon metastables are well known to be very important carriers of energy in argon-containing atmospheric pressure plasma jets [49]. It is reasonable to expect that their concentration would be relatively high, even though the emission spectra do not provide direct evidence. In comparable microwave plasma jets, the argon metastable concentration up to $10^{19} \mathrm{~m}^{-3}$ was reported $[50,51]$.
The atomic oxygen lines observed in this spectral region originate from upper states with energy around $11 \mathrm{eV}$. The lines of oxygen are almost as strong as those of argon and corresponding Einstein emission coefficients are comparable (see table 1), which indicates similar populations of upper states of both atomic oxygen and argon. However, the total concentration of argon inside the discharge tube must exceed the total concentration of atomic oxygen by several orders of magnitude, even if all the water molecules are dissociated, since the water vapour content in the feed gas is only $2600 \mathrm{ppm}$. There are three important processes leading to excitation of atomic oxygen into the ${ }^{3} \mathrm{P}$ and ${ }^{5} \mathrm{P}$ states-(i) direct excitation by electron impact, (ii) a step-wise excitation by electrons via atomic oxygen metastables at 2 and $4 \mathrm{eV}$ and (iii) collisions with argon metastables. Excitation of the $13 \mathrm{eV}$ argon states, on the other hand, arises predominantly due to electron collisions, either from their ground state or via the metastable states at $11.5 \mathrm{eV}$. The excitation by electron impact favours atomic oxygen since its excited states, metastables or the radiative states in question, have much lower energy. Clearly, the amount of electrons with energy of 2 or $4 \mathrm{eV}$ greatly exceeds the amount of those with energy of 11 or $13 \mathrm{eV}^{5}$. Particularly the ${ }^{1} D_{2}$ state of atomic oxygen has a large cross-section for electronic excitation [54]. As was suggested in [55], the chemical kinetics of argon plasma containing water vapour is overly complex. However, the processes described above could, in authors' opinion, explain the observed experimental phenomena.

The emission of $\mathrm{N}_{2}\left(C^{3} \Pi_{u} \rightarrow B^{3} \Pi_{g}\right)$ and $\mathrm{N}_{2}^{+}\left(B^{2} \Sigma_{u}^{+} \rightarrow X^{2} \Sigma_{g}^{+}\right)$ in the spectral region around $355 \mathrm{~nm}$ is the strongest in the discharge plume and is significantly reduced in the afterglow. The decrease of intensity of both systems at the discharge end is probably caused by a combination of two effects. First, part of the nitrogen molecules and molecular ions is dissociated or transformed to other species by chemical processes and second, the available energy could be insufficient to excite nitrogen molecules. It is interesting to note that NO emission is getting stronger further from the discharge tube end. The energy of the relevant vibronic states of NO is only around

5 The excitation temperature calculated from Ar I emission lines, which is believed to be a lower limit for electron temperature, was previously found to be around $4000 \mathrm{~K}$ [42]. The electron density obtained from the Stark broadening of the $\mathrm{H}_{\beta}$ line using Gigosos relation [52] was measured to be in the order of $10^{14} \mathrm{~cm}^{-3}$ [53]. 


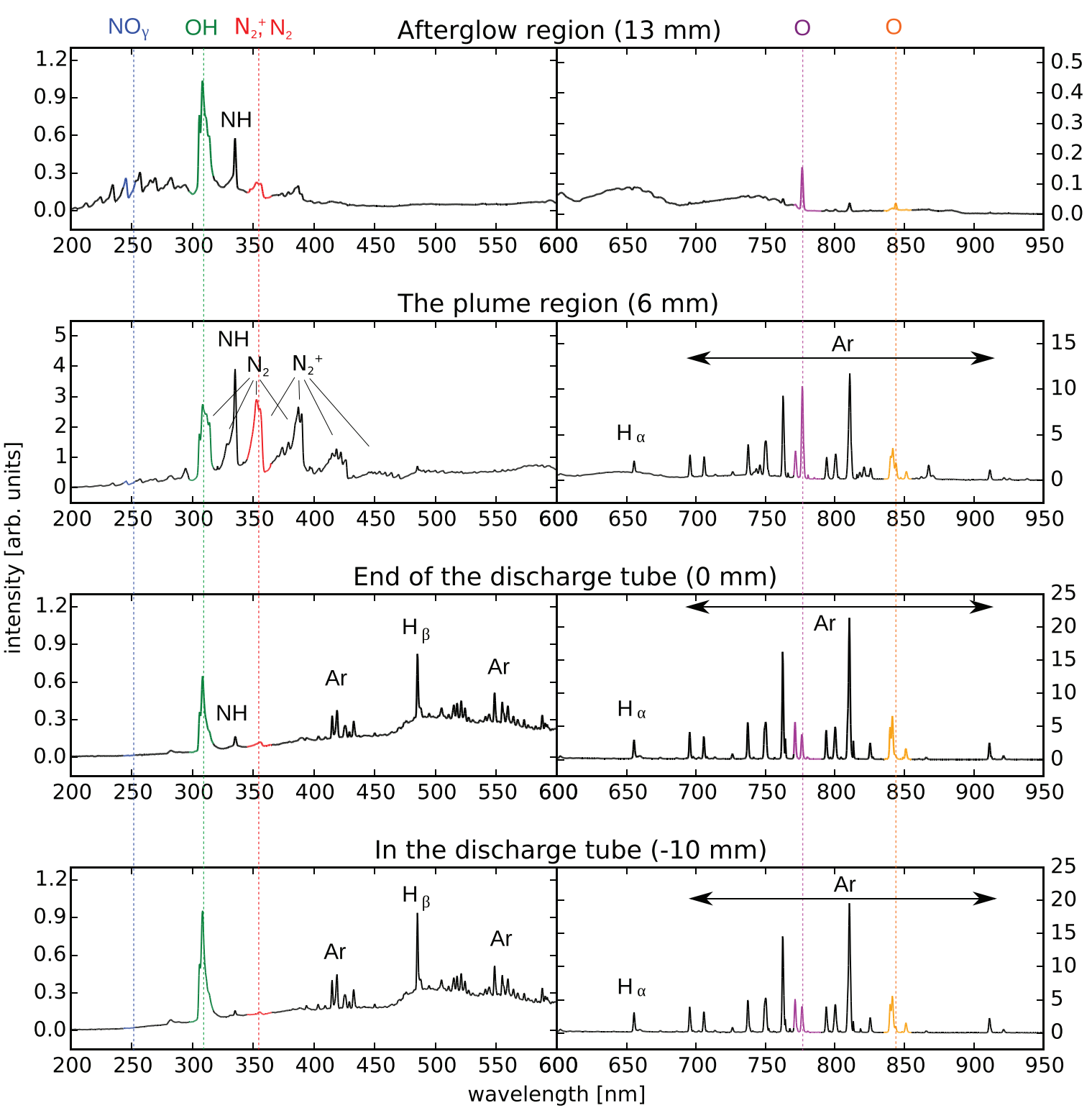

Figure 4. Time-integrated overview spectra at various axial positions of the discharge at modulation frequency of $1710 \mathrm{~Hz}$. The coloured regions correspond to the used bandpass filters listed in section 2.2. Note that the vertical scaling is different in the left and right parts of the plots.

$5 \mathrm{eV}$, while the $\mathrm{N}_{2}\left(C^{3} \Pi_{\mathrm{u}}\right)$ is around $11 \mathrm{eV}[56]$. So the energy insufficient for nitrogen molecule excitation is still sufficient for NO excitation.

The $\mathrm{OH}\left(A^{2} \Sigma \rightarrow X^{2} \Pi\right)$ emission at $307 \mathrm{~nm}$ is always present, as controlled small amount of water was added to the feed gas and water was also present in the ambient air. However, care must be taken when interpreting the signal of $\mathrm{OH}$ emission, because of substantially different quenching rates by the relevant gases. The lifetime of $\mathrm{OH}\left(A^{2} \Sigma^{+}\right)$in pure argon at atmospheric pressure almost equals its natural lifetime of approx. $700 \mathrm{~ns}[32,57]$. Even in argon with water vapour admixture of $2600 \mathrm{ppm}(\mathrm{V})$ the lifetime of $\mathrm{OH}\left(A^{2} \Sigma^{+}\right)$ is still several hundreds of ns. However, in pure air, its lifetime goes down to approx. $2 \mathrm{~ns}$. The expected ratio of the emission intensities from the regions with mostly argon to the emission from regions with mostly air is thus in the order of $10^{2}[32,57]$. So, significantly reduced $\mathrm{OH}$ emission does not necessarily imply reduced $\mathrm{OH}$ concentration. This aspect will be particularly important when discussing the spectrally filtered images later. At this wavelength, also nitrogen $C^{3} \Pi_{\mathrm{u}} \rightarrow B^{3} \Pi_{\mathrm{g}}$ emission is present, namely the bands $(1,0)$ at $315.9 \mathrm{~nm},(2,1)$ at $313.6 \mathrm{~nm},(3,2)$ at $311.7 \mathrm{~nm}$ and $(4,3)$ at $310.4 \mathrm{~nm}$.

\subsection{Spectrally filtered images}

The time-integrated overview spectra taken at four fundamentally different regions of the discharge provided a basic insight into the plasma chemistry, but could not reveal the details of spatial distribution of emission in particular modulation phases. For this, the ICCD camera and bandpass filters had to be employed. Based on the overview spectra, the five bandpass filters mentioned in section 2.2 were selected in order to visualize the plasma-chemical processes and also the gas dynamics in the jet plume, at least partially. 


$\begin{array}{lll}- \text { In the discharge tube }(-10 \mathrm{~mm}) & \text { The plume region }(6 \mathrm{~mm}) \\ -\quad \text { End of the discharge tube }(0 \mathrm{~mm}) & -\quad \text { Afterglow region }(13 \mathrm{~mm})\end{array}$

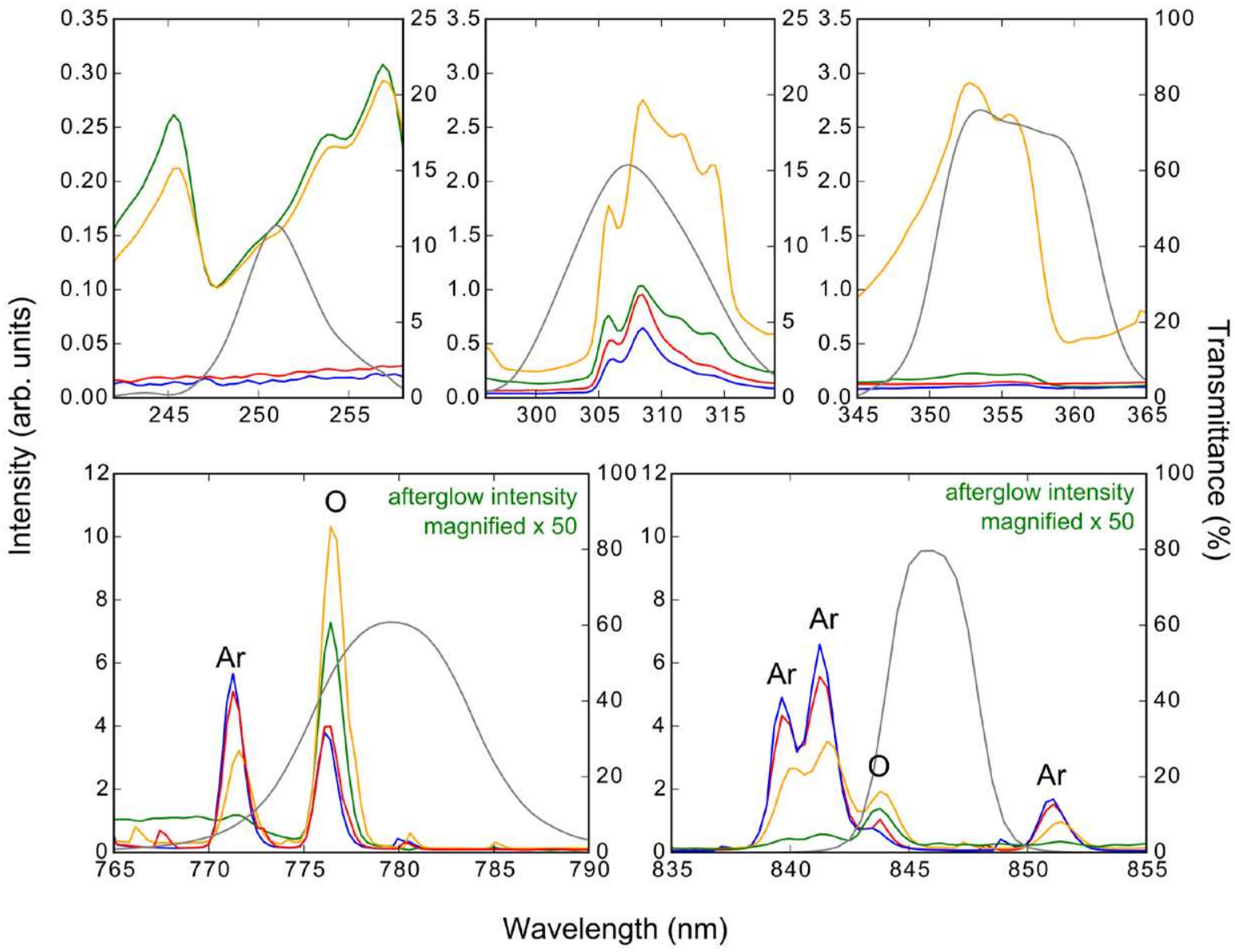

Figure 5. Details of time-integrated spectra in the bandpass-filter regions. The transmittance of the bandpass filters is depicted by the black line. Modulation frequency is $1710 \mathrm{~Hz}$.

Table 1. List of atomic line parameters and properties of corresponding bandpass filters at $780 \mathrm{~nm}$ and $845 \mathrm{~nm}$.

\begin{tabular}{|c|c|c|c|c|c|c|c|c|c|c|c|}
\hline \multirow[b]{2}{*}{ Ion } & \multirow{2}{*}{$\begin{array}{l}\text { Wavelength } \\
(\mathrm{nm})\end{array}$} & \multirow[b]{2}{*}{$\mathrm{A}_{k i}\left(\mathrm{~s}^{-1}\right)$} & \multirow[b]{2}{*}{$\mathrm{E}_{i}(\mathrm{eV})$} & \multirow[b]{2}{*}{$\mathrm{E}_{k}(\mathrm{eV})$} & \multicolumn{3}{|c|}{ Lower Level } & \multicolumn{3}{|c|}{ Upper Level } & \multirow{2}{*}{$\begin{array}{l}\text { Filter } \\
\text { transmittance }\end{array}$} \\
\hline & & & & & Conf. & Term & $\mathrm{J}$ & Conf. & Term & $\mathrm{J}$ & \\
\hline Ar I & 772.37 & $5.18 \times 06$ & 11.548 & 13.153 & $3 s^{2} 3 p^{5}\left({ }^{2} P_{3 / 2}^{\circ}\right) 4 s$ & ${ }^{2}[3 / 2]^{\circ}$ & 2 & $3 s^{2} 3 p^{5}\left({ }^{2} P_{3 / 2}^{\circ}\right) 4 p$ & ${ }^{2}[3 / 2]^{\circ}$ & 1 & $10.87 \%$ \\
\hline Ar I & 772.42 & $1.17 \times 07$ & 11.723 & 13.327 & $3 s^{2} 3 p^{5}\left({ }^{2} P_{1 / 2}^{\circ}\right) 4 s$ & ${ }^{2}[1 / 2]^{\circ}$ & 0 & $3 s^{2} 3 p^{5}\left({ }^{2} \mathrm{P}_{1 / 2}^{\circ}\right) 4 s$ & ${ }^{2}[1 / 2]^{\circ}$ & 1 & $11.06 \%$ \\
\hline O I & 777.19 & $3.69 \times 07$ & 9.146 & 10.740 & $2 s^{2} 2 p^{3}\left({ }^{4} s^{\circ}\right) 3 s$ & ${ }^{5} \mathrm{~S}^{\circ}$ & 2 & $2 s^{2} 2 p^{3}\left({ }^{4} S^{\circ}\right) 3 p$ & ${ }^{5} \mathrm{P}$ & 3 & $52.98 \%$ \\
\hline O I & 777.41 & $3.69 \times 07$ & 9.146 & 10.740 & $2 s^{2} 2 p^{3}\left({ }^{4} S^{\circ}\right) 3 s$ & ${ }^{5} \mathrm{~S}^{\circ}$ & 2 & $2 s^{2} 2 p^{3}\left({ }^{4} S^{\circ}\right) 3 p$ & ${ }^{5} \mathrm{P}$ & 2 & $54.48 \%$ \\
\hline O I & 777.53 & $3.69 \times 07$ & 9.146 & 10.740 & $2 s^{2} 2 p^{3}\left({ }^{4} S^{\circ}\right) 3 s$ & ${ }^{5} \mathrm{~S}^{\circ}$ & 2 & $2 s^{2} 2 p^{3}\left({ }^{4} S^{\circ}\right) 3 p$ & ${ }^{5} \mathrm{P}$ & 1 & $55.25 \%$ \\
\hline Ar I & 842.46 & $2.15 \times 07$ & 11.623 & 13.094 & $3 s^{2} 3 p^{5}\left({ }^{2} P_{3 / 2}^{\circ}\right) 4 s$ & ${ }^{2}[3 / 2]^{\circ}$ & 1 & $3 s^{2} 3 p^{5}\left({ }^{2} P_{3 / 2}^{\circ}\right) 4 p$ & ${ }^{2}[3 / 2]^{\circ}$ & 2 & $3.24 \%$ \\
\hline O I & 844.62 & $3.22 \times 07$ & 9.521 & 10.988 & $2 s^{2} 2 p^{3}\left({ }^{4} S^{\circ}\right) 3 s$ & ${ }^{3} S^{\circ}$ & 1 & $2 s^{2} 2 p^{3}\left({ }^{4} S^{\circ}\right) 3 p$ & ${ }^{3} \mathrm{P}$ & 0 & $66.31 \%$ \\
\hline O I & 844.63 & $3.22 \times 07$ & 9.521 & 10.988 & $2 s^{2} 2 p^{3}\left({ }^{4} S^{\circ}\right) 3 s$ & ${ }^{3} \mathrm{~S}^{\circ}$ & 1 & $2 s^{2} 2 p^{3}\left({ }^{4} S^{\circ}\right) 3 p$ & ${ }^{3} \mathrm{P}$ & 2 & $66.59 \%$ \\
\hline O I & 844.67 & $3.22 \times 07$ & 9.521 & 10.988 & $2 s^{2} 2 p^{3}\left({ }^{4} s^{\circ}\right) 3 s$ & ${ }^{3} \mathrm{~S}^{\circ}$ & 1 & $2 s^{2} 2 p^{3}\left({ }^{4} S^{\circ}\right) 3 p$ & ${ }^{3} \mathrm{P}$ & 1 & $67.61 \%$ \\
\hline Ar I & 852.14 & $1.39 \times 07$ & 11.828 & 13.283 & $3 \mathrm{~s}^{2} 3 \mathrm{p}^{5}\left({ }^{2} \mathrm{P}_{1 / 2}^{\circ}\right) 4 \mathrm{~s}$ & ${ }^{2}[1 / 2]^{\circ}$ & 1 & $3 s^{2} 3 p^{5}\left({ }^{2} P_{1 / 2}^{\circ}\right) 4 p$ & ${ }^{2}[3 / 2]^{\circ}$ & 2 & $0.10 \%$ \\
\hline
\end{tabular}

Note: Einstein coefficients $\mathrm{A}_{k i}$, energies $\mathrm{E}_{k}$ and $\mathrm{E}_{i}$ of upper and lower levels, respectively, and other constants are taken from [56]. 

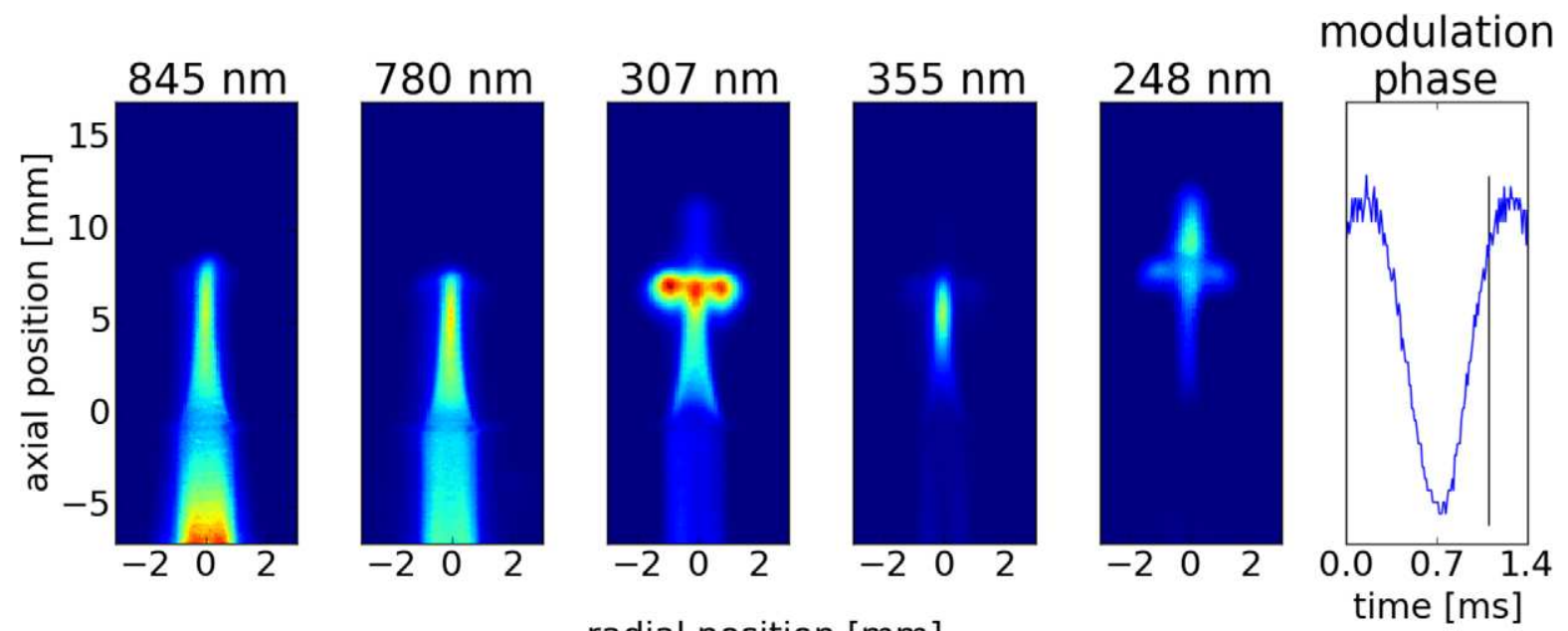

radial position $[\mathrm{mm}]$

Figure 6. Images of the surfatron plasma jet modulated at $900 \mathrm{~Hz}$ taken through pre-selected bandpass filters with 200 shots averaging. Single shot exposure time was $10 \mu \mathrm{s}$. The images are in false colours and scaling is different for each image. The last panel depicts modulation of the measured microwave power with the black vertical line indicating the phase, at which the images were taken.

A typical set of plasma jet images taken through the five bandpass filters is shown in figure 6 . These images were taken during the rising part of the sine envelope of the power modulation at $900 \mathrm{~Hz}$ modulation frequency, as indicated in the rightmost plot.

The filters with central wavelength of 780 and $845 \mathrm{~nm}$ transmit mostly the radiation of excited argon atoms, thus indicating the regions of nearly unperturbed plasma originally ignited in the feed gas. As seen in figure 6, the emission at 845 and $780 \mathrm{~nm}$ takes place mainly in the discharge tube and in the lower part of the jet plume. Unfortunately, the oxygen atoms also emit in both spectral regions. The atomic oxygen is present partly due to dissociation of the water vapour introduced into the feed gas, but also due to mixing of plasma with the ambient air.

The filter centred at $307 \mathrm{~nm}$ transmits the emission of $\mathrm{OH}$ radicals and vibrationally excited $\mathrm{N}_{2}$ molecules. The transmittance profile of the filter clearly favours the higher vibrational levels of $\mathrm{N}_{2}$. Together with the quenching of $\mathrm{OH}$ mentioned above, this brings us to the following interpretation of the signal collected through the $307 \mathrm{~nm}$ filter. This spontaneous plasma emission visualizes the regions where nitrogen molecules appear in small quantities with high excitation degree and high vibrational temperature and also where $\mathrm{OH}$ is readily formed and excited, presumably by dissociation of water by collisions with highly energetic electrons and argon metastables. In other words, this visualizes the region where ambient air comes to the first contact with the highly energetic plasma plume. In figure 6 , around $7 \mathrm{~mm}$ from the discharge tube end a sharp increase of emission at $307 \mathrm{~nm}$ appears. Its spatial distribution reveals a remarkable structure at this axial distance, which is presumably caused by a flow perturbation and resembles a toroidal vorte ${ }^{6}$. The vortex was moving upwards in time

\footnotetext{
${ }^{6}$ Most of our results suggest a presence of a vortex-like structure. The techniques used in this work, however, do not enable to conclusively prove a presence of a vortex. Nevertheless, for the sake of brevity, we sometimes refer to this flow perturbation as a 'vortex' instead of a more appropriate 'zone of enhanced gas mixing' further in the text.
}

and reappearing at each modulation period in the rising slope of power. The temporal evolution of the emission at $307 \mathrm{~nm}$ in the effluent, showing the ascent of the vortex, can be seen in figure 7. The motion of the flow perturbation and related phenomena will be discussed in more detail later. Emission at $307 \mathrm{~nm}$ is also present in the whole active plasma, but since the feed gas contained only a small amount of water vapour, the $\mathrm{OH}$ emission in the discharge tube is relatively weak.

The filter centred at $355 \mathrm{~nm}$ transmits mainly the emission of excited molecules of $\mathrm{N}_{2}$ and $\mathrm{N}_{2}^{+}$. This emission is a good indicator of strong admixture of ambient atmosphere into the active plasma, since no nitrogen was added into the feed gas. The emission of nitrogen molecule and molecular ion in figure 6 appears at the current position of the vortex but also behind it, i.e. at the places of previous vortex locations. This indicates very efficient mixing of the plasma plume with the surrounding air.

The filter centred at $248 \mathrm{~nm}$ transmits mostly the emission of NO. In all observed cases, this emission appears further along the plasma plume-above the vortex-and indicates the chemistry induced by admixing the air into the active plasma.

The spontaneous emission patterns partly reveal the fluid dynamics. It is observed that during the part of the modulation period when the driving power is rising, a zone of enhanced mixing of the plasma plume and the surrounding air appears. It could be concluded, that the rapid expansion of the plasma plume, presumably accompanied by heating of the gas flowing through the discharge tube, leads to formation of a transient flow perturbation, most likely a toroidal vortex. Inside the vortex, the surrounding air flows directly into the active plasma. As a result, the air constituents, together with the inherent water vapour, can be excited and dissociated, leading to the spontaneous emission patterns observed.

3.2.1. Processed streak-like images. The set of images in figure 6 was only the typical example of the instantaneous situation at given modulation phase. Similar image sets were recorded using variable trigger delay, effectively scanning 

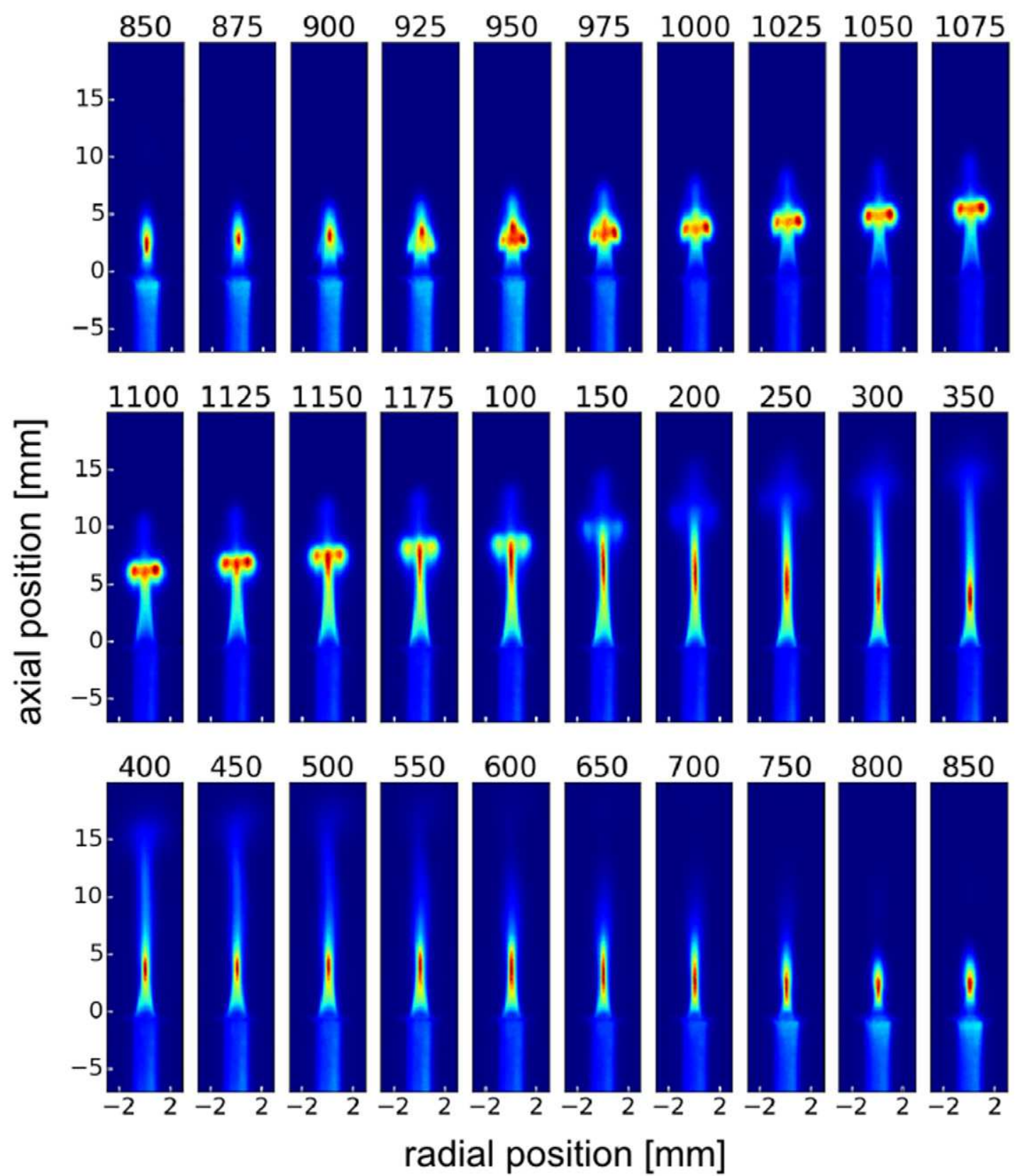

Figure 7. Temporal evolution of emission at $307 \mathrm{~nm}$ during a single period at $900 \mathrm{~Hz}$ power modulation. The image titles show the delay (in microseconds) of the ICCD camera trigger compared to the power modulating signal. The delays can be directly compared to those in figure 2 .

over the whole power modulation period, as shown in figure 7. To provide more concise insight, the data were processed into a streak-like images in the following way. Each image was integrated horizontally, resulting in a one-dimensional array, containing the information about the axial distribution of emission intensity. This was performed for each measured delay over the power modulation period and plotted as a streak-like image as illustrated in figure 8 . The processed results may be found in figure 9, showing the spatio-temporal evolution of the relative emission intensity for each spectral filter, at modulation frequencies 90, 900 and $1710 \mathrm{~Hz}$. For each frequency, the time scale was adjusted to show slightly more than one full period.

As stated above, the spectral vicinity of the argon and oxygen lines at the wavelength regions of the bandpass filters centred at $780 \mathrm{~nm}$ and $845 \mathrm{~nm}$ does not allow to reliably distinguish the emission of oxygen and argon atoms. Nevertheless, as shown in figure 5, it seems reasonable to attribute most of the signal passing through the filter centred at $845 \mathrm{~nm}$ to the oxygen atoms. In figure 9, a distant maximum of the emission at $845 \mathrm{~nm}$ can be observed at the end of the visible plasma plume, which spatially coincides with the emission of nitrogen molecules. This indicates that the admixture of air provides more oxygen molecules for dissociation and excitation by collisions with the active plasma constituents. Interestingly, the strongest signal is observed inside the discharge tube from the positions closest to the surfatron cavity, even though the amount of oxygen is expected to be significantly smaller compared to the effluent. From the available data it is not possible to decide whether this is solely due to oxygen atoms emission, 


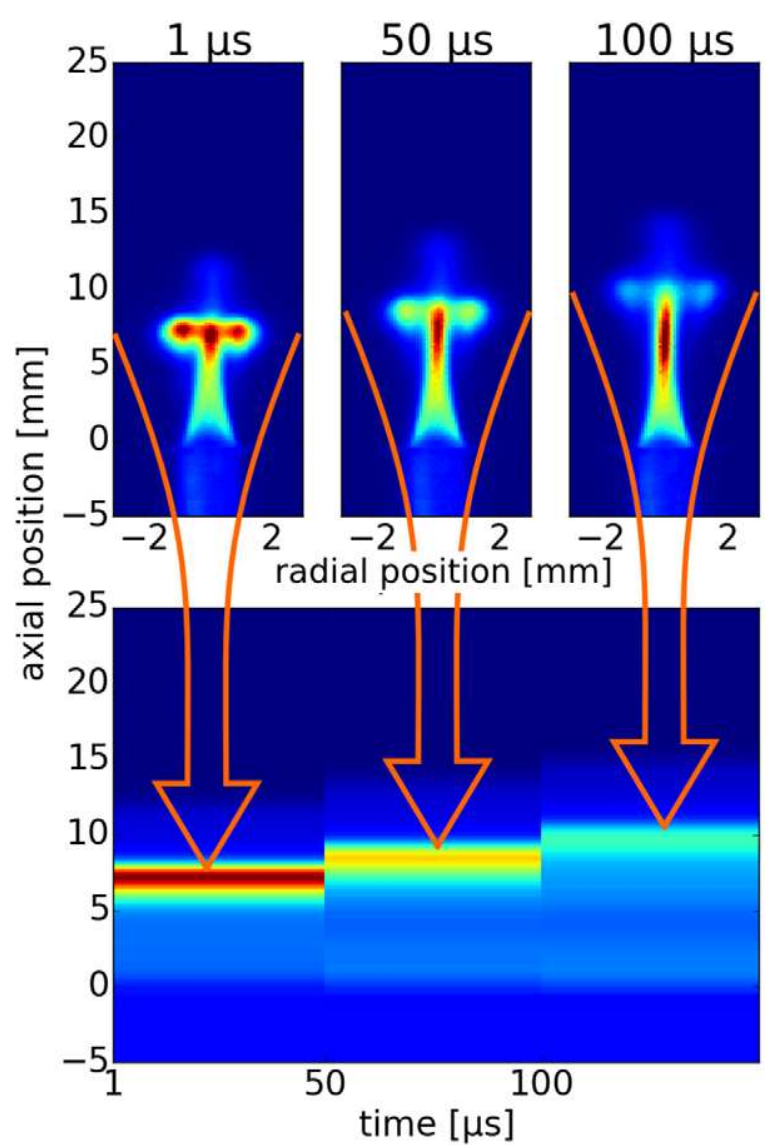

Figure 8. Illustration of the data processing described in section 3.2.1. The upper images are transformed into vertical lines by integration along the horizontal axis.

or if the argon emission is so strong that it compensates for the lower transmittance of the bandpass filter.

The spatio-temporal pattern of the emission at $780 \mathrm{~nm}$ is quite similar to that at $845 \mathrm{~nm}$. The lower and the upper maximum is present in both cases. However, in direct comparison, the pattern at $780 \mathrm{~nm}$ seems to be slightly flatter. The oxygen emission at $777.4 \mathrm{~nm}$ originates in the state with energy $10.74 \mathrm{eV}$ and that at $844.6 \mathrm{~nm}$ originates at $10.99 \mathrm{eV}$. These are indeed very close and the spatio-temporal patterns of both spectral emissions are expected to be very similar at both wavelengths. The energies of the respective argon upper states are even closer. What is expected to differ between $845 \mathrm{~nm}$ and $780 \mathrm{~nm}$ spectral ranges is the ratio of argon to oxygen emission, favouring argon at $780 \mathrm{~nm}$. Thus, it may be concluded that the axial profile of the argon emission is rather flat or at least falls monotonously towards the end of the visible discharge. Time integrated measurements of argon emission point rather to the latter. This would be also in accordance with the fact that the microwave power dissipation density is the highest in the vicinity of the surfatron launching gap, as was shown e.g. in $[47,58]$.

The emission at $307 \mathrm{~nm}$ depicts nicely most of the relevant features of the gas dynamics in the effluent. For modulations at $900 \mathrm{~Hz}$ and $1710 \mathrm{~Hz}$, a vortex structure is formed. Due to the horizontal integration and the enhanced emission of $\mathrm{N}_{2}$, the vortex structure introduces a sharp maximum into the streak-like images, see figure 9. Note that the shown images are averaged over 200 shots. The formation and the location of the vortex is thus remarkably reproducible. The location of the maximum tracks the upwards movement of the vortex. It can be seen that the vortex is clearly observable during the part of the modulation period when the plasma effluent is elongating. After that, the rising vortex loses contact with the active plasma region and gradually fades as the excited species are depleted.

The image for $90 \mathrm{~Hz}$ is though quite different from those of higher frequencies. The vortex maximum is not present for this frequency and the $\mathrm{OH}$ emission is distributed much more evenly through the whole length of the discharge. The $\mathrm{OH}$ emission might appear as generally stronger for this frequency, but it is only the outcome of smaller intensity range-see the colour scaling. The absence of the vortex for modulation frequency of $90 \mathrm{~Hz}$ indicates that the modulation of the power at this frequency may not have been fast enough to create the flow instability leading to the vortex formation.

For higher frequencies, the axial location of the sharp maximum at $307 \mathrm{~nm}$, indicating the vortex, foreruns and partially coincides with the emission of nitrogen molecule and molecular ion at $355 \mathrm{~nm}$. The vortex enhances the mixing of the cooling air layer and possibly also the ambient air with the active plasma. Figure 9 suggests that in case of $90 \mathrm{~Hz}$ modulation (which corresponds to $11.1 \mathrm{~ms}$ period) $\mathrm{N}_{2}$ and $\mathrm{N}_{2}^{+}$ bands appears in the plasma plume as result of outer gas diffusion. For higher frequencies of 900 and $1710 \mathrm{~Hz}(1.1$ and $0.6 \mathrm{~ms}$ respective periods) due to the short modulation period the vortex mixing appears as main mechanism of species exchange [59] between feed and cooling gas as gas diffusion is insufficient.

The emission of NO appears above the vortex, i.e. downstream. As the appearance of NO emission is accompanied by the disappearance of the $\mathrm{OH}$ emission and decrease of emission of $\mathrm{N}_{2}$ and $\mathrm{N}_{2}^{+}$, it might be tempting to interpret this as a chemical reaction converting nitrogen and $\mathrm{OH}$ to NO. Such reaction was described in the reference [60] as existing but extremely slow. The disappearance of $\mathrm{OH}$ emission is caused rather by the quenching of $\mathrm{OH}\left(A^{2} \Sigma^{+}\right)$by admixed air, as discussed above. The reduced emission of nitrogen cannot be explained solely by quenching. For example, the rate of quenching of $\mathrm{N}_{2}^{+}\left(B^{2} \Sigma_{u}^{+}\right)$by air constituents is in the same order as the rate of quenching by argon [61]. Nevertheless, it is reasonable to assume that the rapid admixing of ambient air leads to rapid decrease of the electron and excitation temperatures. This would certainly suppress the emission of ions and neutrals with highly energetic upper state such as $\mathrm{N}_{2}\left(C^{3} \Pi_{\mathrm{u}}\right)$. As discussed above, the excitation energy of NO is much lower and the emission still appears readily above the vortex. This gives us a clue about the excitation temperature above the vortex. Even after admixing cool ambient air, the plasma contains enough energy to excite $5 \mathrm{eV}$ states.

Interestingly, the NO emission intensity is not significantly weaker for the $90 \mathrm{~Hz}$ modulation, where no vortex formation was observed. From the available data, it is impossible to decide if this is due to the fact that the total amount of 
(a)
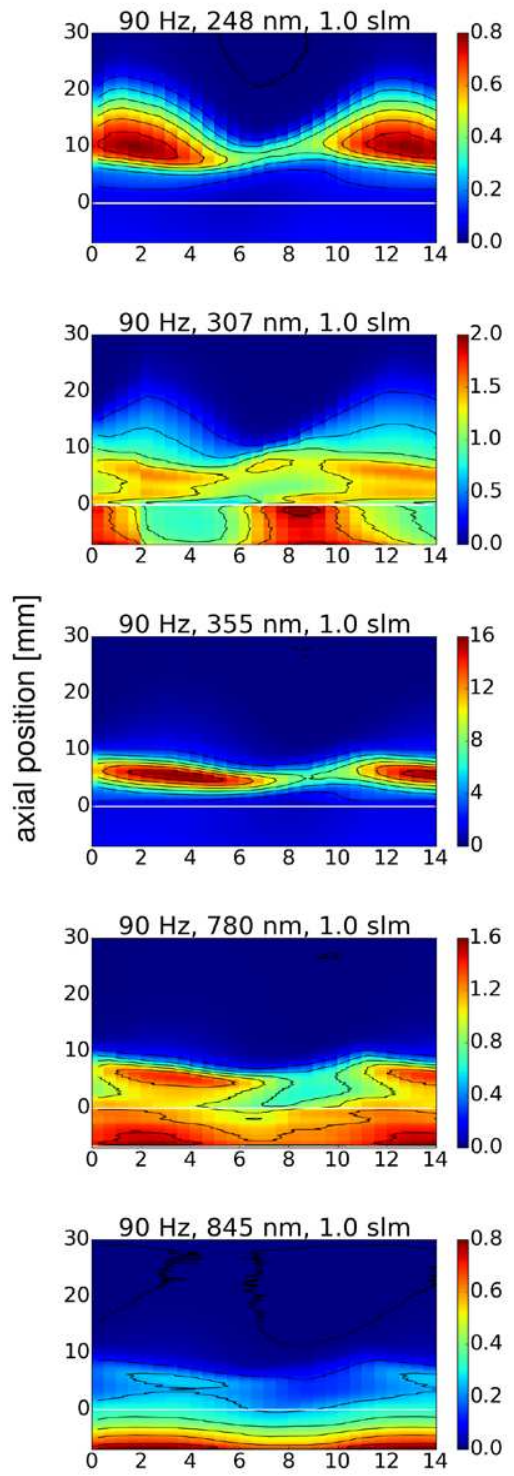

(b)
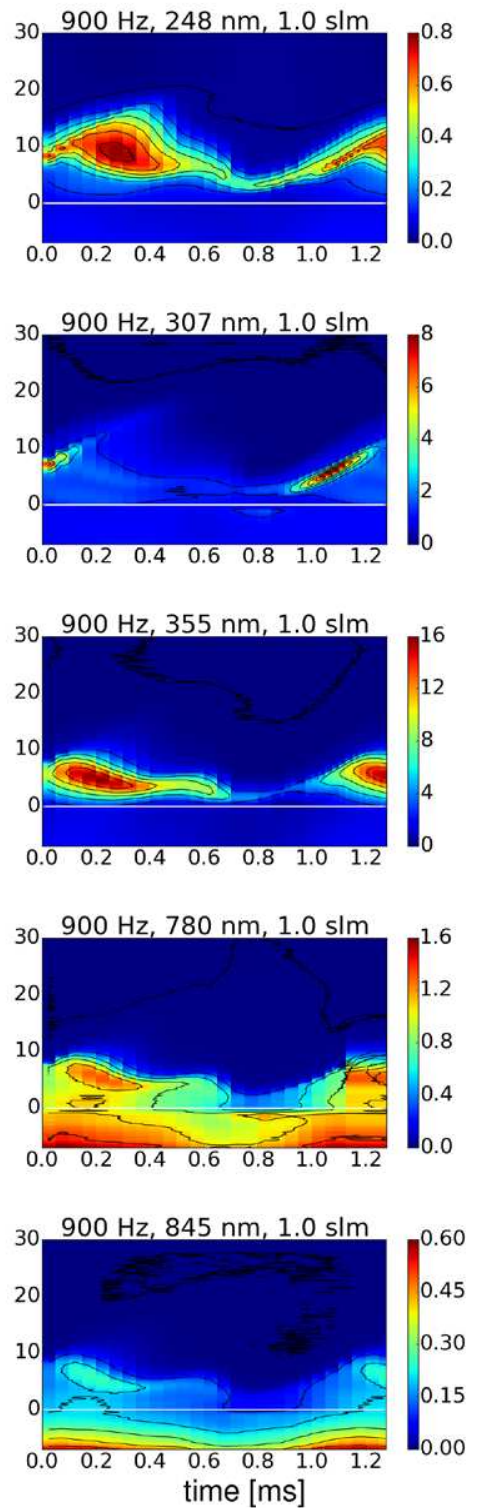

(c)
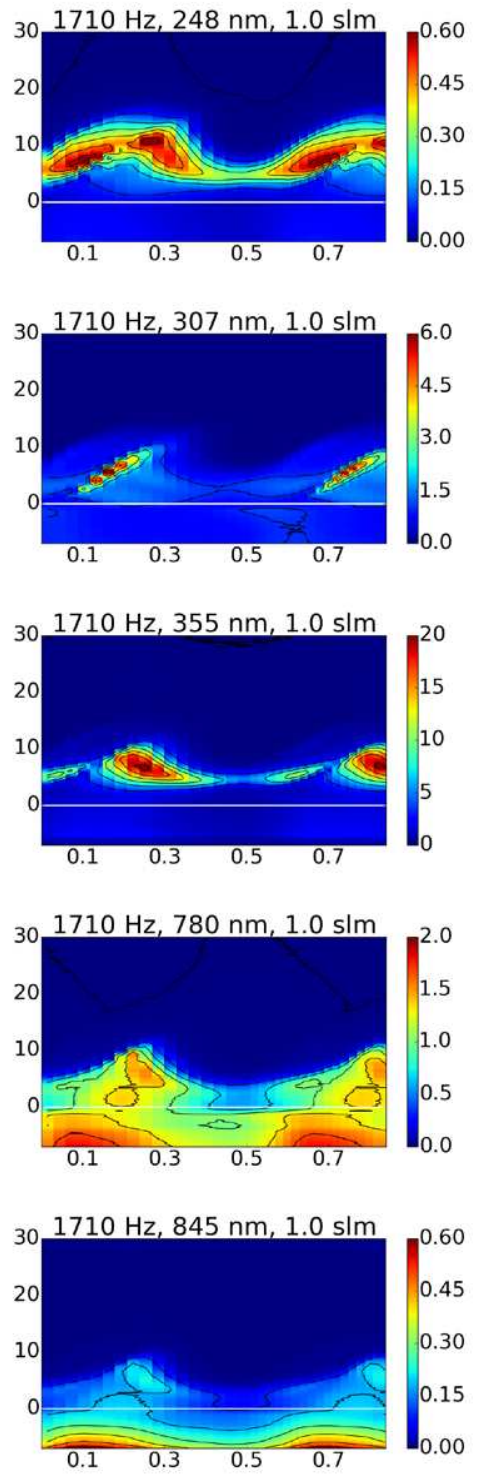

Figure 9. Streak-like depiction of evolution of laterally integrated emission intensity during modulation period for the five spectral regions. The discharge tube end is indicated by a white horizontal line at axial position of $0 \mathrm{~mm}$. The argon flow rate in all cases was $1.0 \mathrm{slm}$. The modulation frequency was (a) $90 \mathrm{~Hz}$, (b) $900 \mathrm{~Hz}$ and (c) $1710 \mathrm{~Hz}$.
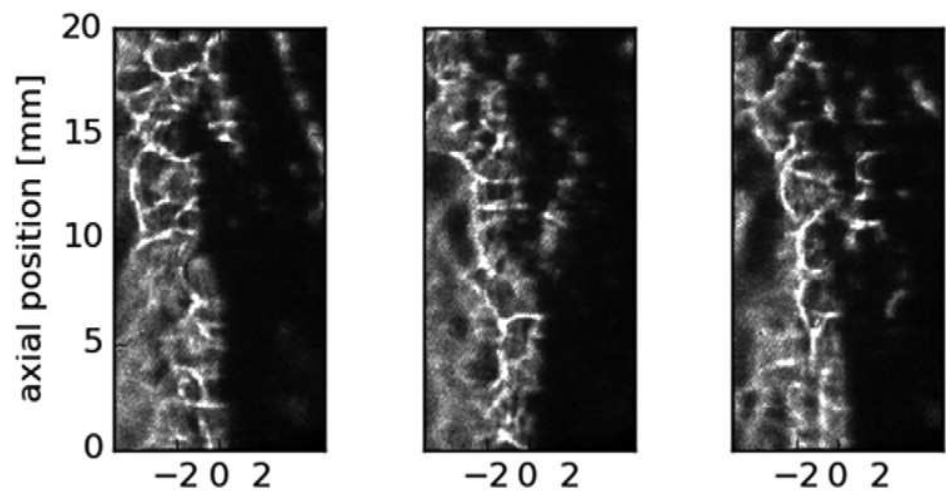

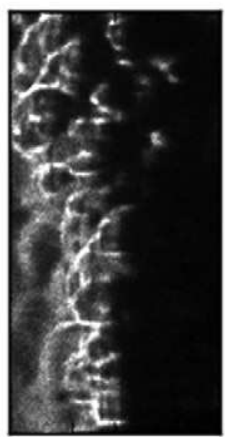

$-202$

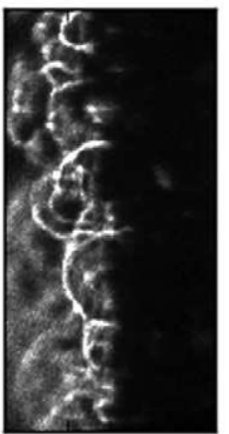

$-202$ radial position $[\mathrm{mm}]$

Figure 10. Single-shot schlieren images of the plasma plume at modulation frequency of $900 \mathrm{~Hz}$ for various modulation phases. The exposure time was $4 \mu \mathrm{s}$. No underlying structure is visible due to highly stochastic character. 

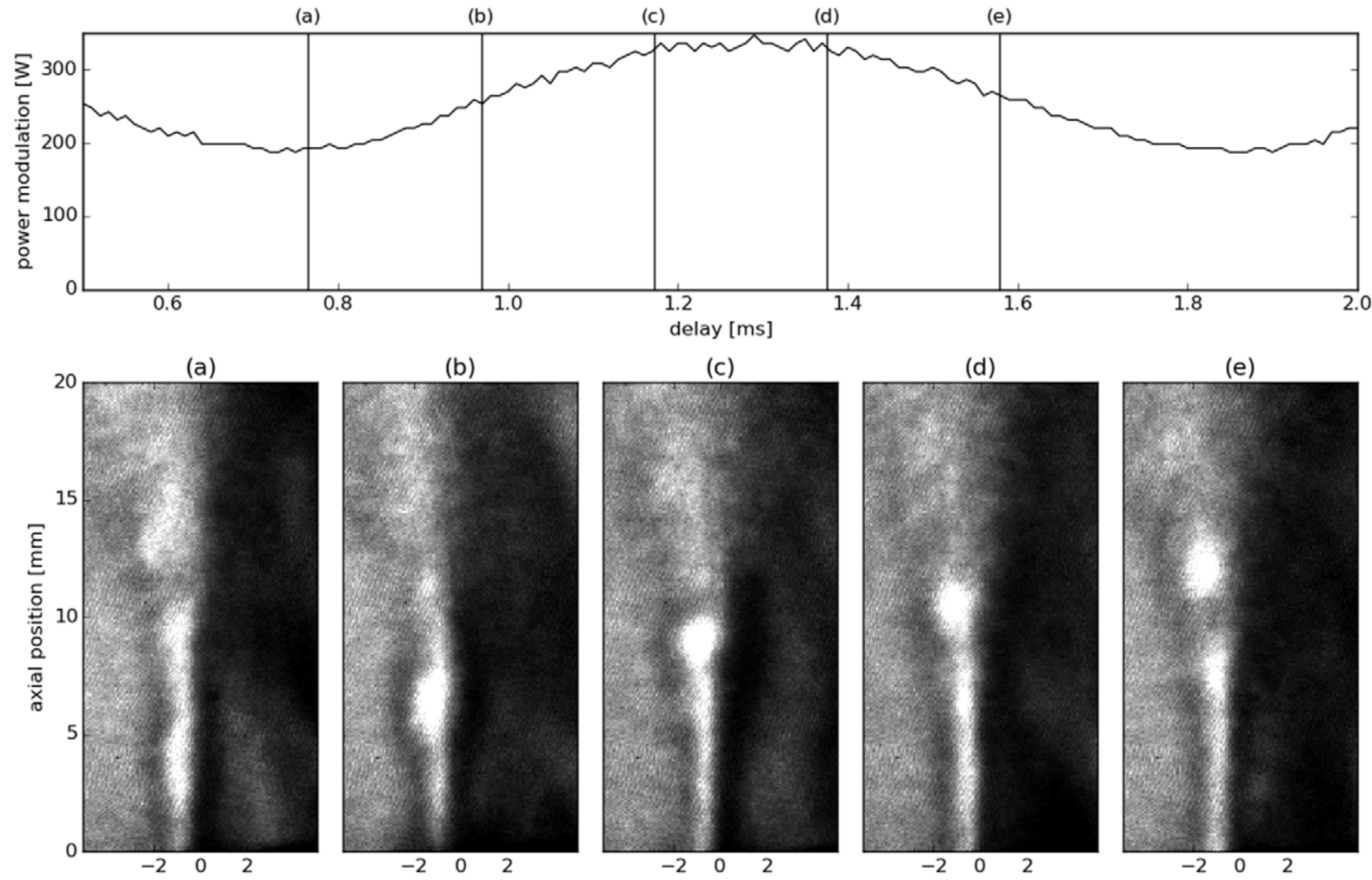

(b)

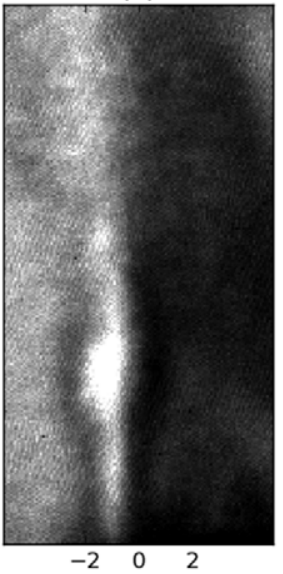

(c)

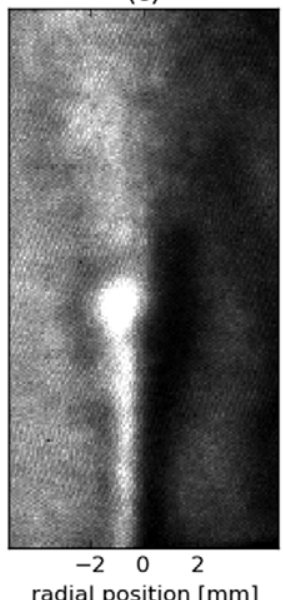

(d)

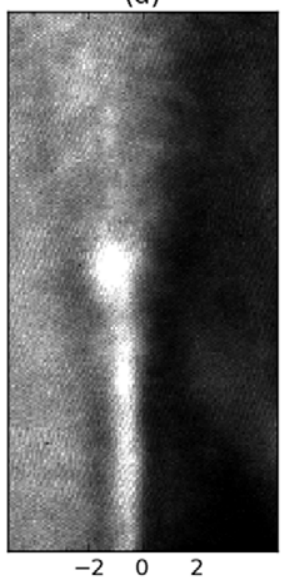

(e)

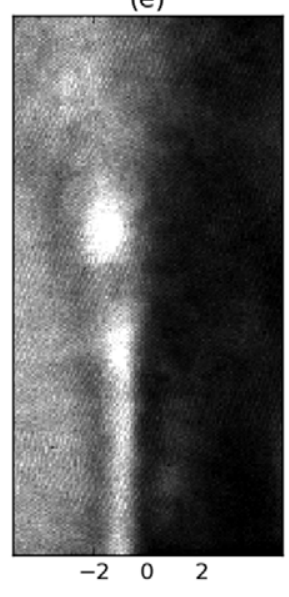

Figure 11. Schlieren images averaged over 100 periods of the discharge power modulation. The exposure time was $100 \times 4 \mu$ s. The power modulation curve is depicted above, the delays of the individual images are shown by the vertical lines.

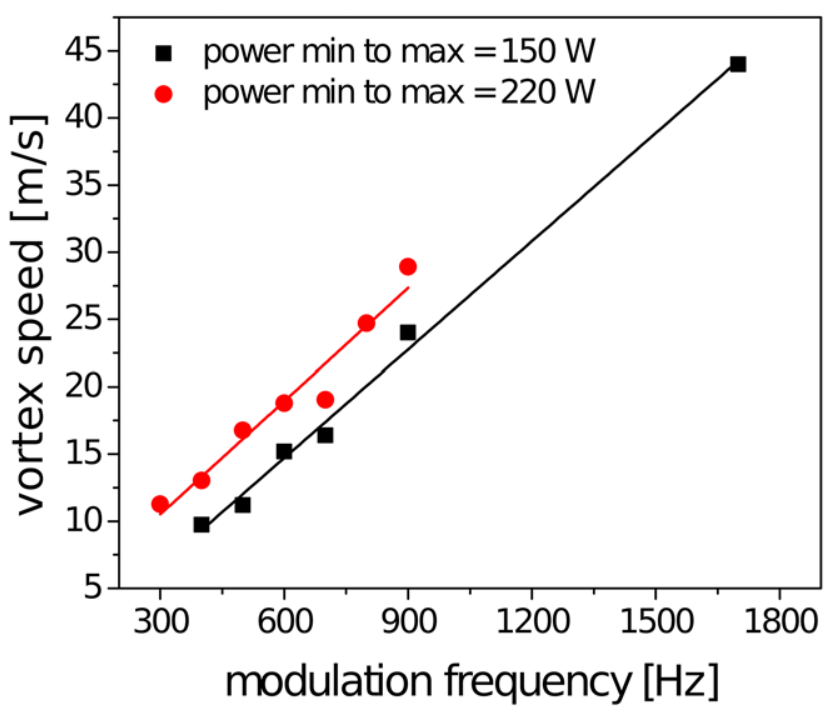

Figure 12. The speed of the upwards vortex motion as a function of the modulation frequency for two sine modulation envelope power ranges: (i) black squares- $\min =165 \mathrm{~W}$, $\max =315 \mathrm{~W}$, difference $=150 \mathrm{~W}$; (ii) red circles- $\min =155 \mathrm{~W}$, $\max =375 \mathrm{~W}$, difference $=220 \mathrm{~W}$.

NO is not significantly affected by the vortex formation, or rather that the temperature drop due to the vortex compensates for enhanced NO production, leading to weaker excitation degree. Although the authors are inclined rather to the latter explanation, temporally and spatially resolved thermometry and absorption or laser-induced fluorescence measurement of NO density would be necessary to validate this hypothesis.

\subsection{Phase-resolved schlieren images of the plasma plume}

Even though the spatio-temporal patterns of the spontaneous plasma emission strongly suggest that a transient vortex is formed, the fluid dynamics should be examined with a more straightforward method. The schlieren photography was selected as it is highly sensitive, easy to carry out and well respected in gas flow studies [21, 26, 29, 62]. The first singleshot images revealed the chaotic behaviour of the interface between the hot and flowing cooling air (encompassing the plasma plume) and the static cold ambient air, see figure 10 . Unfortunately, the stochastically varying refractive index gradients at this interface were sufficient to hide any eventual flow structures on the interface between the cooling air and the plasma plume.

Taking a series of images averaged over 100 periods of the power modulation helped to suppress the chaotic effects on the outer interface and to highlight the reproducible flow patterns on the inner interface, see figure 11. The blurring is an unavoidable consequence of the temporal averaging. Note that the spontaneous plasma emission is not present in the images due to arrangement of the imaging optics and the use of the interference filter as described in section 2. The schlieren contrast patterns spatially and temporally coincide with the patterns of the spontaneous plasma emission (comp. figures 11 and 7) and further support the transient vortex hypothesis. It also appears that the vortex moves further upwards when it loses contact with the active plasma region, as could have been expected but could not be observed in the spontaneous emission images. 

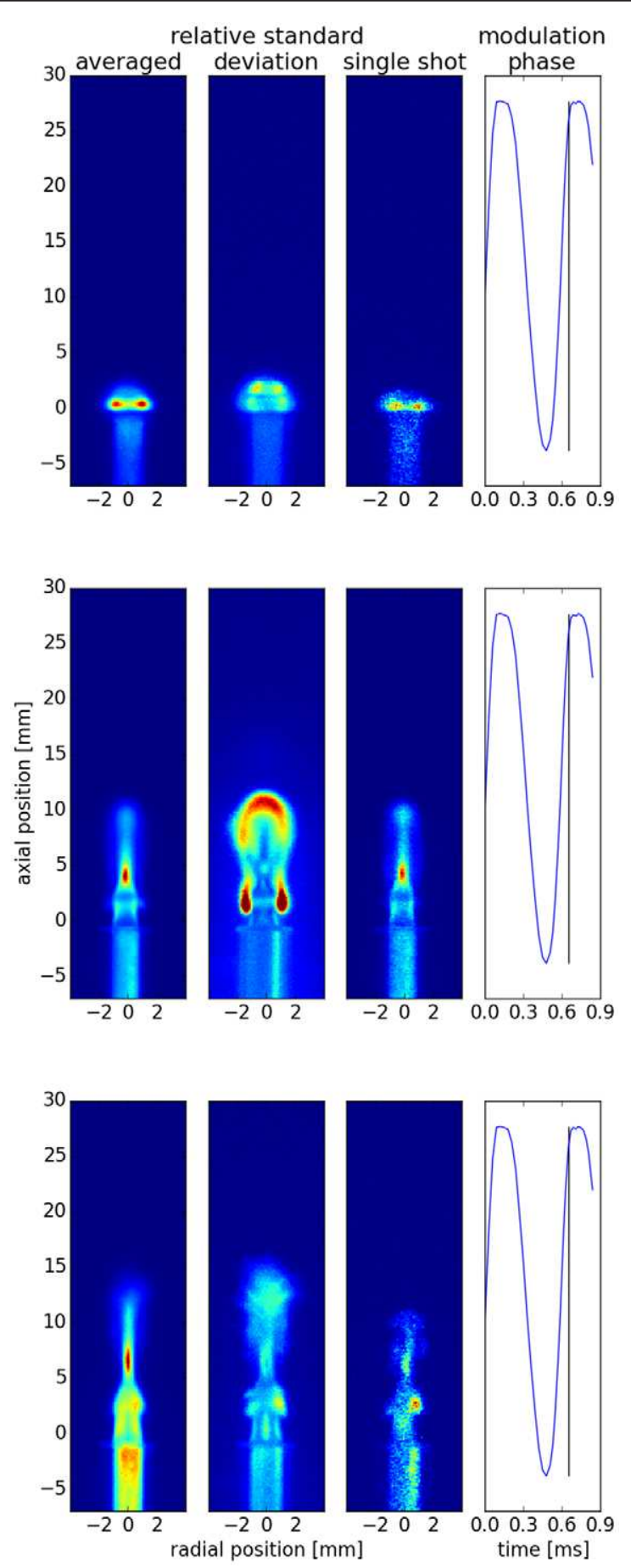

Figure 13. Comparison of emission at $307 \mathrm{~nm}$ for flow rates of $0.5 \mathrm{slm}, 1 \mathrm{slm}$ and $2 \mathrm{slm}$, from top to bottom, respectively.

It should be emphasised that the mechanism behind the eventual vortex formation is still unclear and it would require further studies to fully explain the gas dynamics in the effluent. This, however, is beyond the scope of this work.

\subsection{Influence of experimental parameters on vortex formation and propagation}

Further attention has been paid to the analysis of the vortex, which is presumably the most important feature discovered by the performed imaging, as it significantly influences the
Table 2. The speed of vortex motion in metres per second.

\begin{tabular}{llll}
\hline & $900 \mathrm{~Hz}$ & $1710 \mathrm{~Hz}$ & $\begin{array}{l}\text { Expected } \\
\text { flow speed }\end{array}$ \\
\hline $0.5 \mathrm{slm}$ & 19 & 33 & 22 \\
$1.0 \mathrm{slm}$ & 24 & 44 & 44 \\
$2.0 \mathrm{slm}$ & N/A & 53 & 88 \\
\hline
\end{tabular}

Note: The vortex was not sufficiently reproducible for $900 \mathrm{~Hz}$ modulation and $2.0 \mathrm{slm}$ flow.

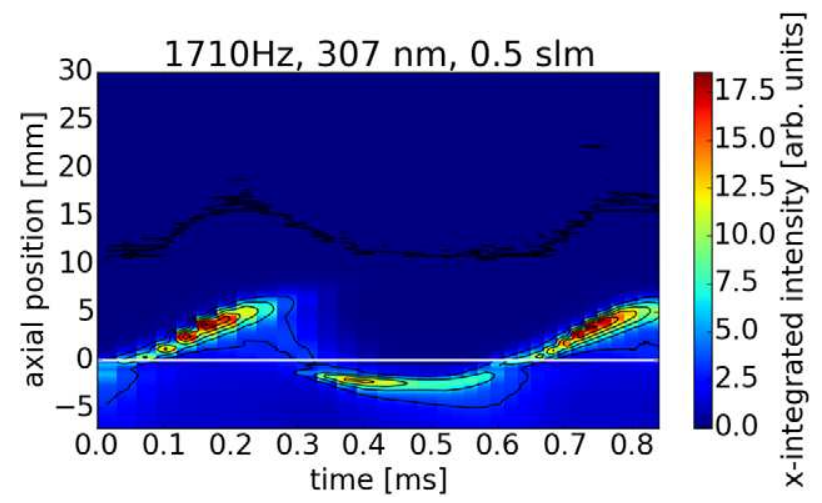

Figure 14. Streak-like image for power modulation at $1710 \mathrm{~Hz}$ and reduced feed gas flow rate of $0.5 \mathrm{slm}$.

plasma chemistry in the effluent. To study the vortex, several experimental parameters (modulation frequency, feed gas flow rate, modulation envelope) were varied and the discharge was observed through the bandpass filter at $307 \mathrm{~nm}$, as vortexlike structure is the most noticeable on the spatial patterns of this emission.

3.4.1. Modulation frequency. As already said, the vortex has been observed for higher modulation frequencies, but not for the $90 \mathrm{~Hz}$ frequency. No matter if it is due to the too slow change in power or due to insufficiently rapid plasma elongation, the modulation frequency clearly is an important parameter for the vortex formation. However, no sharp lower boundary, or minimum modulation frequency at which the vortex would be formed, was found. For low frequencies, the presence or absence of the vortex were rather occasional-it was formed only at the fraction of periods. Also, it was not quite spatially stable and was of low intensity. Such unstable vortex is not observable in averaged images and can only be noticed at single shot images. As the modulation frequency increased, the formation of the vortex became gradually more and more regular.

The slew rate $\mathrm{d} P / \mathrm{d} t$, i.e. the change in instantaneous power per time interval is probably the key parameter determined by the modulation frequency. When the modulation frequency is increased, the sine modulation envelope at fixed power settings gets steeper and the corresponding change in power happens in shorter time. The other way of controlling the power change per time is to adjust the power settings at given modulation frequency. In all previous measurements, the instantaneous power oscillated between $165 \mathrm{~W}$ in minimum and $315 \mathrm{~W}$ in maximum - the minimum to maximum power difference was $150 \mathrm{~W}$. For the next experiment, this power difference was 

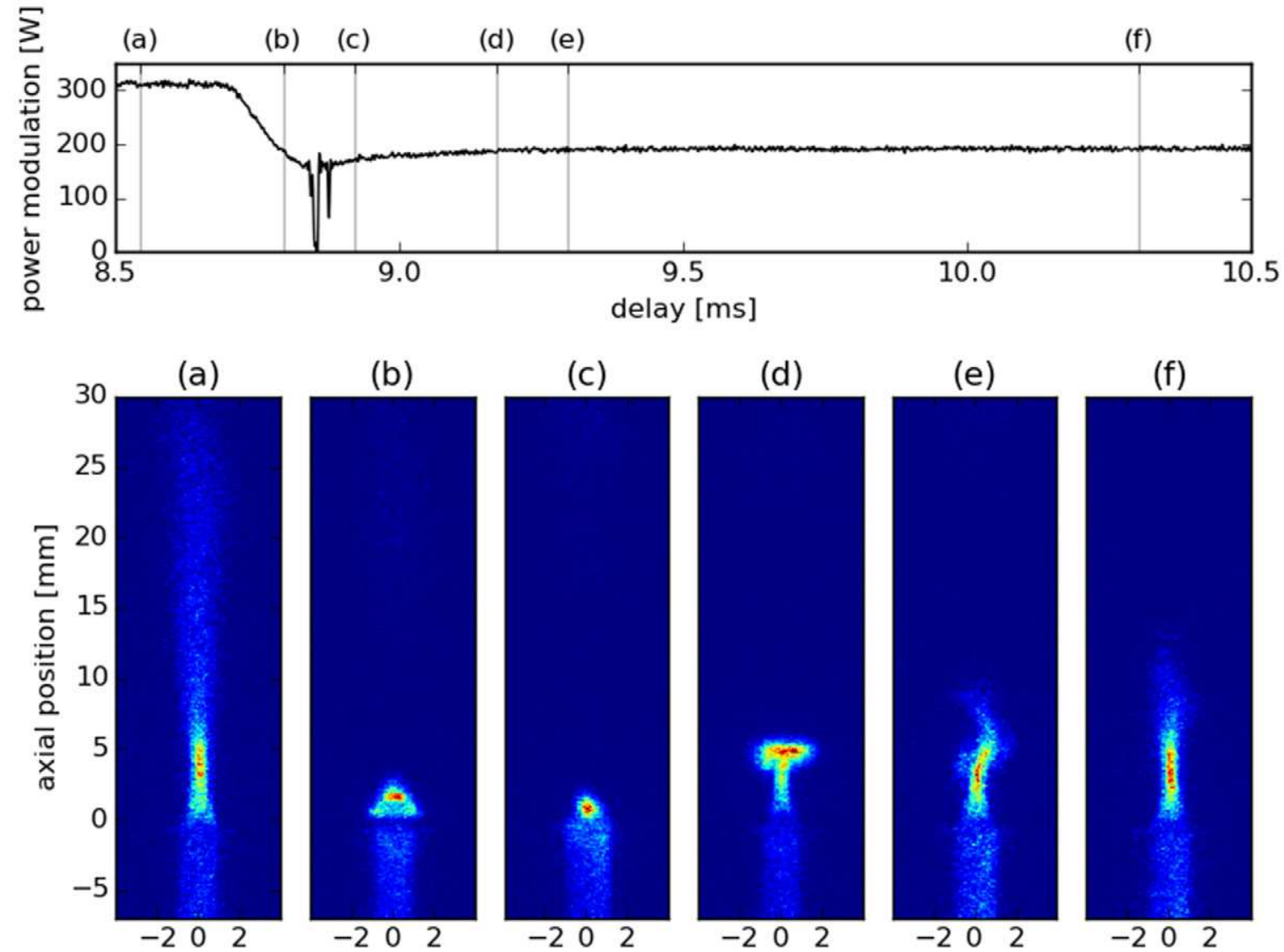

(b)

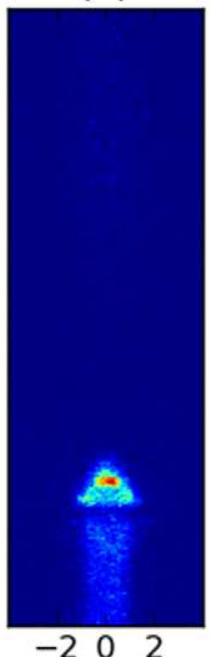

(c)

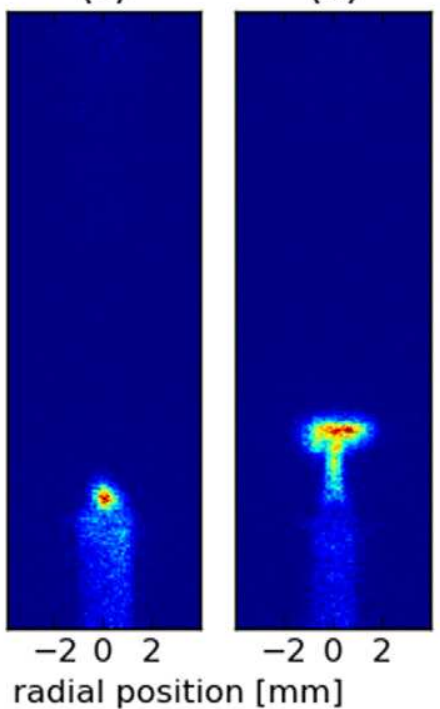

(e)

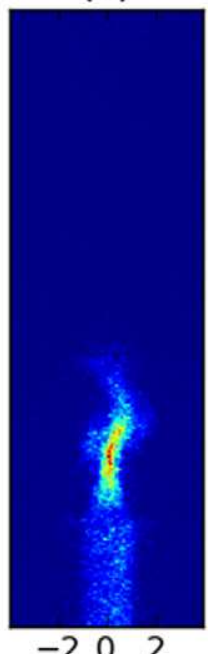

(f)

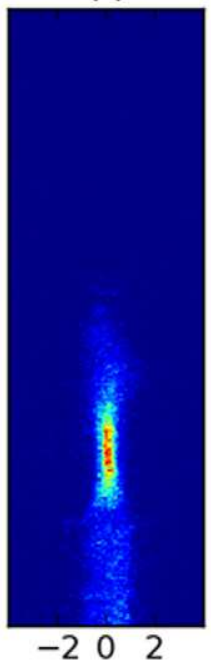

Figure 15. Single shot images of the discharge through $307 \mathrm{~nm}$ filter (OH emission) revealing the evolution of the effluent at the falling edge of the rectangular power modulation envelope $(60 \mathrm{~Hz}$ modulation frequency). The images (a-f) show the plasma emission in different phases, see the upper image for time reference.

increased to $220 \mathrm{~W}$, with $155 \mathrm{~W}$ in minimum and $375 \mathrm{~W}$ in maximum. The observation of the vortex formation at these altered conditions proved that the same relative regularity and stability of the vortex was observed at lower modulation frequencies than in the previous case.

For further comparison of the two modulation amplitude cases, the speed of the vortex upwards motion was calculated. From the results in figure 12 it can be seen that the vortex speed depends linearly on the modulation frequency for both modulation amplitudes. If the speed of the vortex had been determined predominantly by the power change per time, the slope of the trend would have been higher for higher power difference. However, the trends diverge only insignificantly, which is rather unexpected.

3.4.2. Feed gas flow rate. Another parameter that intuitively should be influencing the speed of the vortex, is the feed gas flow rate. To investigate this, the measurements were performed for the 'normal' sine modulation (165 W-315W) at argon flow rates of $0.5 \mathrm{slm}$ and $2 \mathrm{slm}$, i.e. one half and twice of the original value of $1 \mathrm{slm}$. The discharge images of the emission at $307 \mathrm{~nm}$ for the three gas flow rates at the same modulation phase can be seen in figure 13. The speeds of the vortex ascents, under the well justified assumption of entirely linear motion, are listed in table 2 . For comparison, the expected speed of the argon flow in the discharge tube is also given, assuming idealized case with constant temperature of $2500 \mathrm{~K}$. This value is based on the measurement of rotational temperature of the $\mathrm{OH}$ radical. This temperature, often expected to be identical with the temperature of the neutral gas, was found to vary with the phase, but not significantly [43].

The fact that the vortex upwards speed perfectly matches the speed of the actively radiating plasma elongation during the rise of the modulated microwave power should not be surprising, since the plasma emission patterns are governed by the gas mixing as discussed above. The speeds given in table 2 suggest that the the gas flow rate is not the main factor affecting the speed of the vortex ascent.

The streak-like image for the lowest feed gas flow rate of $0.5 \mathrm{slm}$ and highest modulation frequency of $1710 \mathrm{~Hz}$ revealed another interesting feature, see figure 14. The sharp maximum of $\mathrm{OH}$ emission moves inside the discharge tube for the part of the modulation period with the lowest power. As verified by the nitrogen emission (not shown for brevity), this is caused by introduction of the ambient air into the discharge tube. The cooling of the discharge caused by the lack of microwave power was rapid enough to temporarily reverse the gas flow. This verifies the speculation about the negative flow velocity in [42]. 
3.4.3. Modulation envelope. When the modulation envelope was changed from sine to rectangular, a flow reversal was observed, too. Rectangular envelope dictates very fast power transitions. In idealized case instantaneous, while in real world the slew rate is limited by the equipment imperfections. From oscilloscopic measurements the slope of power change with time when using $60 \mathrm{~Hz}$ rectangular modulation corresponds to a maximum slope achieved with sine modulation frequency of approx. $2700 \mathrm{~Hz}$. The power absorbed in the discharge is not determined only by the incident power coming from the microwave generator, but also by the instantaneous plasma properties. These properties (shape, electron density, temperatures, etc) influence the plasma impedance. The change of the plasma properties can be significantly delayed and/or distorted with respect to the modulated power output of the generator.

Nevertheless, the rectangular envelope offers very fast power changes and it is then not surprising that the vortex formation at the power rising edge was detected for all rectangular modulation frequencies. Just like for the sine modulation, the vortex was not formed at falling edge of modulation. Instead, more complex effect was observed (see figure 15). After the drop of the instantaneous power, the plasma began to shrink rapidly, see figure 15 (b). For most of the constant lower part of the rectangular envelope the effluent length was fixed at certain value $l$, see figure 15(f). However, for few moments just after the sharp decrease of power, its length reached even shorter minimum $l^{\prime}<l$, (figures 15(b) and (c)), followed closely by the fast expansion to the length $l$ (figure 15(d)). This behaviour is probably caused by the local pressure decrease after rapid cooling of gas and enhanced further by the undesired and unintended, though extremely short break in generator output power. In some cases it was accompanied by a reverse flow, i.e. suction of the air into the discharge tube observed as $\mathrm{OH}$ emission maximum inside (figure 15(b)), (just like in figure 14) and the consequent expansion also gave a rise to a vortex, visible in figure $15(\mathrm{~d})$.

\section{Conclusion}

The spectrally and temporally resolved imaging of power modulated microwave surfatron plasma jet operated at atmospheric pressure argon was performed. The obtained image series enabled qualitative analysis of the plasma-chemical processes in the effluent.

For higher modulation frequencies a formation of highly reproducible transient flow perturbation resembling a vortex was observed. This perturbation was observed on the plasma emission spatial patterns as well as on the schlieren images. The vortex was moving upwards, in the flow direction, but with a speed different from the expected gas flow speed. Furthermore, the speed of the vortex ascent was found to depend only weakly on the flow rate of the feed gas. On the other hand, the vortex speed changed proportionally to the frequency of the sine power modulation. Further detailed studies are needed to explain this behaviour.

The presence of the flow perturbation strongly influences the plasma-chemical and flow processes in the effluent. It also leads to effective broadening of the active plasma region, resulting in better homogeneity in a wider cross-sectional area. This feature may be advantageously utilized for optimisation of the plasma jet for desired applications.

The gas dynamics was found to be very strongly influenced by thermal effects of the power-modulated discharge. At certain conditions a local flow reversal leading to suction of ambient atmosphere into the discharge tube end was observed.

\section{Acknowledgment}

The authors would like to thank D Nečas for measurements of transmittance of the bandpass filters and P Konečný for help with the schlieren photography. The graphs in this publication were composed in the Matplotlib 2D graphics environment [63]. The information on chemical kinetics were found in NIST chemical kinetics database [64]. The information about the spectroscopic quantities, including the energy of atomic levels, were found in NIST atomic spectra database [56].

This research has been supported by the project CZ.1.05/2.1.00/03.0086 funded by European Regional Development Fund and project LO1411 (NPU I) funded by Ministry of Education Youth and Sports of Czech Republic and 'CEITEC - Central European Institute of Technology' (CZ.1.05/1.1.00/02.0068) from the European Regional Development Fund and by 'Research center of surface treatment', project TE02000011 of Technology Agency of the Czech Republic. Petr Synek would like to acknowledge the project 'Employment of Newly Graduated Doctors of Science for Scientific Excellence' (CZ.1.07/2.3.00/30.0009) co-financed from European Social Fund and from the national budget of the Czech Republic.

\section{References}

[1] Wagner H E, Brandenburg R, Kozlov K, Sonnenfeld A, Michel P and Behnke J 2003 Vacuum 71 417-36

[2] Kogelschatz U 2003 Plasma Chem. Plasma Process. 23 1-46

[3] Tendero C, Tixier C, Tristant P, Desmaison J and Leprince P 2006 Spectrochim. Acta B 61 2-30

[4] Laroussi M and Akan T 2007 Plasma Process. Polym. $4777-88$

[5] Merche D, Vandencasteele N and Reniers F 2012 Thin Solid Films 520 4219-36

[6] Synek P, Jašek O, Zajiččková L, David B, Kudrle V and Pizúrová N 2011 Mater. Lett. 65 982-4

[7] Belmonte T, Arnoult G, Henrion G and Gries T 2011 J. Phys. D: Appl. Phys. 44363001

[8] Jimenez M, Rincon R, Marinas A and Calzada M 2013 Int. J. Hydrog. Energy 38 8708-19

[9] Hnilica J, Schäfer J, Foest R, Zajíčková L and Kudrle V 2013 J. Phys. D: Appl. Phys. 46335202

[10] Kong M G, Kroesen G, Morfill G, Nosenko T, Shimizu T, Van Dijk J and Zimmermann J 2009 New J. Phys. 11115012

[11] Laroussi M 2009 IEEE Trans. Plasma Sci. 37 714-25

[12] Ehlbeck J, Schnabel U, Polak M, Winter J, Von Woedtke T, Brandenburg R, Von dem Hagen T and Weltmann K 2011 J. Phys. D: Appl. Phys. 44013002 
[13] Weltmann K D, Kindel E, Brandenburg R, Meyer C, Bussiahn R, Wilke C and Von Woedtke T 2009 Contrib. Plasma Phys. 49 631-40

[14] Heinlin J, Isbary G, Stolz W, Morfill G, Landthaler M, Shimizu T, Steffes B, Nosenko T, Zimmermann J and Karrer S 2011 J. Eur. Acad. Dermatology Venereology $251-11$

[15] Potočňáková L, Hnilica J and Kudrle V 2015 Open Chem. 13 541-8

[16] Schütze A, Jeong J Y, Babayan S E, Park J, Selwyn G S and Hicks R F 1998 IEEE Trans. Plasma Sci. 26 1685-94

[17] Walsh J L, Iza F, Janson N B, Law V and Kong M G 2010 J. Phys. D: Appl. Phys. 43075201

[18] Lu X, Laroussi M and Puech V 2012 Plasma Sources Sci. Technol. 21034005

[19] Schäfer J, Foest R, Ohl A and Weltmann K 2009 Plasma Phys. Control. Fusion $\mathbf{5 1} 124045$

[20] Hnilica J, Kudrle V, Vašina P, Schäfer J and Aubrecht V 2012 J. Phys. D: Appl. Phys. $\mathbf{4 5} 055201$

[21] Robert E, Sarron V, Darny T, Riès D, Dozias S, Fontane J, Joly L and Pouvesle J M 2014 Plasma Sources Sci. Technol. 23012003

[22] Zhang S, Sobota A, van Veldhuizen E and Bruggeman P 2015 J. Phys. D: Appl. Phys. 48015203

[23] Xiong R, Xiong Q, Nikiforov A Y, Vanraes P and Leys C 2012 J. Appl. Phys. 112033305

[24] Shao X J, Chang Z S, Mu H B, Liao W L and Zhang G J 2013 IEEE Trans. Plasma Sci. 41 899-906

[25] Chen Z, Yin Z, Chen M, Hong L, Xia G, Hu Y, Huang Y, Liu M and Kudryavtsev A 2014 J. Appl. Phys. 116153303

[26] Schmidt-Bleker A, Reuter S and Weltmann K 2015 J. Phys. D: Appl. Phys. 48175202

[27] Synek P, Obrusník A, Hübner S, Nijdam S and Zajíčková L 2015 Plasma Sources Sci. Technol. 24025030

[28] Teschke M, Kedzierski J, Finantu-Dinu E, Korzec D and Engemann J 2005 IEEE Trans. Plasma Sci. 33 310-1

[29] Oh J S, Olabanji O T, Hale C, Mariani R, Kontis K and Bradley J W 2011 J. Phys. D: Appl. Phys. 44155206

[30] Papadopoulos P, Vafeas P, Svarnas P, Gazeli K, Hatzikonstantinou P, Gkelios A and Clement F 2014 J. Phys. D: Appl. Phys. 47425203

[31] Iseni S, Schmidt-Bleker A, Winter J, Weltmann K and Reuter S 2014 J. Phys. D: Appl. Phys. 47152001

[32] Voráč J, Obrusník A, Procházka V, Dvořák P and Talába M 2014 Plasma Sources Sci. Technol. 23025011

[33] Boswell R and Henry D 1985 Appl. Phys. Lett. 47 1095-7

[34] Charles C, Boswell R and Kuwahara H 1995 Appl. Phys. Lett. 67 40-2

[35] Ashida S, Lee C and Lieberman M 1995 J. Vac. Sci. Technol. A 13 2498-507

[36] Lieberman M A and Ashida S 1996 Plasma Sources Sci. Technol. 5145

[37] Kelly S and Turner M 2014 Plasma Sources Sci. Technol. 23065012

[38] Takahashi K, Hori M and Goto T 1993 Japan. J. Appl. Phys. 32 L1088
[39] Watanabe Y, Shiratani M, Kubo Y, Ogawa I and Ogi S 1988 Appl. Phys. Lett. 53 1263-5

[40] Courteille C, Dorier J, Hollenstein C, Sansonnens L and Howling A 1996 Plasma Sources Sci. Technol. 5210

[41] Walsh J L, Shi J and Kong M G 2006 Appl. Phys. Lett. 88171501

[42] Hnilica J and Kudrle V 2014 J. Phys. D: Appl. Phys. 47085204

[43] Hnilica J, Potočňáková L and Kudrle V 2015 Open Chem. 13 577-85

[44] Voráč J, Hnilica J, Kudrle V and Dvořák P 2015 Open Chem. 13 193-7

[45] Potočňáková L, Hnilica J and Kudrle V 2013 Int. J. Adhes. Adhes. 45 125-31

[46] Hnilica J, Potočňáková L, Stupavská M and Kudrle V 2014 Appl. Surf. Sci. 288 251-7

[47] Moisan M, Beaudry C and Lepprince P 1974 Phys. Lett. A 50125

[48] Boffard J B, Jung R, Lin C C and Wendt A 2009 Plasma Sources Sci. Technol. 18035017

[49] Santiago I and Calzada M D 2009 IEEE Trans. Plasma Sci. 37 790-6

[50] Timmermans E, Van de Sande M and Van der Mullen J 2003 Plasma Sources Sci. Technol. 12324

[51] Castaños-Martínez E and Moisan M 2010 Spectrochim. Acta B 65 199-209

[52] Gigosos M A, González M Á and Cardeñoso V 2003 Spectrochim. Acta B 58 1489-504

[53] Hnilica J 2013 Time resolved diagnostics of microwave pulsed plasma PhD Thesis Masaryk university, Brno http://is.muni. cz/www/2560/42839596/PhD_thesis_hnilica.pdf

[54] Biagi-v8.9 2015 (Magboltz version 8.9) and Morgan (Kinema Research Software) retrieved on July 7 www.lxcat.net

[55] Van Gaens W and Bogaerts A 2013 J. Phys. D: Appl. Phys. 46275201

[56] Ralchenko Y and Reader J 2015 NIST atomic spectra database (version 5.2) http://physics.nist.gov/asd

[57] Bülter A, Lenhard U, Rahmann U, Kohse-Höinghaus K and Brockhinke A 2004 Laskin: Efficient simulation of spectra affected by energy transfer Proc. of LACEA 2004 Laser Applications to Chemical and Environmentel Analysis

[58] Baeva M, Bösel A, Ehlbeck J and Loffhagen D 2012 Phys. Rev. E 85056404

[59] Chen R H and Driscoll J F 1989 The role of the recirculation vortex in improving fuel-air mixing within swirling flames Symp. (Int.) on Combustion vol 22 (Amsterdam: Elsevier) pp 531-40

[60] Bowman C T 1971 Combust. Sci. Technol. 3 37-45

[61] Freeman C and Phillips L 1974 Can. J. Chem. 52 426-8

[62] Xu D, Shneider M, Lacoste D and Laux C 2014 J. Phys. D: Appl. Phys. 47235202

[63] Hunter J D 2007 Comput. Sci. Eng. 9 90-5

[64] Manion J A et al 2015 NIST chemical kinetics database, NIST standard reference database 17, version 7.0 (web version), release 1.6.8, data version $2013.03 \mathrm{http}: / /$ kinetics.nist.gov/ 